\title{
A Multilevel Monte Carlo Evolutionary Algorithm for Robust Aerodynamic Shape Design
}

\author{
M. Pisaroni*, F. Nobile ${ }^{\dagger}$ and P. Leyland ${ }^{\ddagger}$ \\ Ecole Polytechnique Fédérale de Lausanne (EPFL), CH-1015 Lausanne, Switzerland
}

\begin{abstract}
The majority of problems in aircraft production and operation require decisions made in the presence of uncertainty. For this reason aerodynamic designs obtained with traditional deterministic optimization techniques seeking only optimality in a specific set of conditions may have very poor off-design performances or may even be unreliable. In this work we present a novel approach for robust optimization of aerodynamic shapes based on the combination of single and multi-objective Evolutionary Algorithms and a Continuation Multi Level Monte Carlo methodology to estimate robust designs, without relying on derivatives and meta-models. Detailed numerical studies are presented for a transonic airfoil design affected by geometrical and operational uncertainties.
\end{abstract}

\section{Introduction}

Optimization has always been an integral part in aerodynamic design. Nowadays the ever-increasing demand for aircrafts with better performance, higher reliability and robustness at lower cost requires optimization techniques seeking optimality under uncertain conditions that may arise during design, manufacture and operation of the vehicle. Indeed, the geometrical and operational parameters, that characterize aerodynamic systems, are naturally affected by aleatory uncertainties due to the intrinsic variability of the manufacturing processes and the surrounding environment.

Reducing the geometrical uncertainties due to manufacturing tolerances can be prohibitively expensive while reducing the operational uncertainties due to atmospheric turbulence in external aerodynamics is simply impossible. Optimization under uncertainty (OUU) refers to broad class of methodologies that address mainly the following two problems:

- Robust Design Optimization (RDO): focuses on the performances of a system under perturbations of the design conditions. Prescribed probabilistic measures of robustness (involving mean, variance or higher moments) as objective functions are used to robustify the design. The optimal design should, in this framework, be as much insensitive as possible to uncertain conditions meaning that its performance should not drop below a prescribed quality level.

- Reliability-based Design Optimization (RBDO): focus on safety-under-uncertainty aspects of the system and deals generally with the optimization of a deterministic objective functions subject to probabilistic constraints (failure probability or reliability index). The optimal design has, in this framework, a higher degree of confidence and guarantees a prescribed minimum level of reliability under uncertain conditions.

In this work we concentrate on the former class of problems in aerodynamic shape design. To be relevant for complex industrial engineering problems we also consider designs that require a robust optimization of different and competing objectives: Multi-Objective Robust Design Optimization (MO-RDO).

In this work we consider transonic airfoils under geometric uncertainties due to manufacturing tolerances and operating uncertainties due to intrinsic variability of the surrounding flow. In transonic airfoil RDO we not only wish to minimize shock drag losses at a given speed, as in deterministic optimization, but we

*PhD. Student, Scientific Computing and Uncertainty Quantification Group (CSQI), AIAA Student Member.

$\dagger$ Professor, Head of CADMOS Chair of Scientific Computing and Uncertainty Quantification (CSQI).

${ }^{\ddagger}$ Group Leader, Interdisciplinary Aerodynamics Group (IAG), AIAA Member. 
require that the drag and its dispersion (e.g. standard deviation of the drag) are minimized under a set of geometric and operating uncertain conditions. Supercritical airfoils have been investigated since many years and are usually conceived to operate efficiently with a substantial portion of supersonic flow on the airfoil while maximizing the lift in the aft portion where the flow is subsonic. Although several of these supercritical sections appeared promising during the preliminary design phase, they led to serious problems when incorporated into an aircraft because of their sensitivity to Mach number. As a result they may poorly perform or may be even potentially worthless at off-design conditions leading to the appearance of the so called drag creep, a situation in which substantial section drag increase with Mach number even at speeds below the design value. ${ }^{1}$

Despite the wide availability of modern parallel computer architectures and efficient Computational Fluid Dynamics (CFD) solvers, the key difficulties in RDO are:

- computing the objective functions that involve robust measures based on mean, variances and higher moments when many sources of uncertainties are present (efficient propagation of uncertainties).

- dealing with competing objectives to compute a robust Pareto-optimal front.

Many non-intrusive uncertainty propagation techniques and derivatives-free approaches have gained a lot of interest in recent years as they simply require multiple solutions of the original model and can use available industrial CFD solvers as a black box. Polynomial Chaos (PC) ${ }^{2-4}$ and Stochastic Collocation $(\mathrm{SC})^{5}$ methods have been successfully employed in different engineering fields to built response surfaces of the stochastic variables, while Kriging regression ${ }^{6}$ has been mainly employed to build meta-models of the design variables. All these approaches have a strong potential for RDO but they are known to suffer the so called 'curse of dimensionality' (dramatically increase of computational cost with the number of uncertain/design variables). In addition, those approaches based on global basis functions that span the entire stochastic domain may see their performances and accuracy jeopardized if the problem under investigation present strong discontinuities as in the case of transonic/supersonic flows.

On the other hand Monte Carlo (MC) methods have a dimension independent convergent rate and have been proven to be robust and accurate for non smooth problems, nevertheless their very slow convergence rate makes them chimerical for practical applications. The Multi Level Monte Carlo (MLMC) method has been introduced by Heinrich ${ }^{7}$ and thereupon extended by Giles $^{8}$ to approximate stochastic differential equations (SDEs). The key idea of MLMC is that one can draw MC samples simultaneously and independently on several approximations of the problem under investigations on a hierarchy of nested computational grids (levels). The expectation of a quantity of interest (QoI) is computed as a sample average of coarsest solutions corrected by averages of the differences of the solutions of two consecutive grids in the hierarchy. By this way, most of the computational effort is transported from the finest level to the coarsest one.

The robustness and accuracy of the classical MLMC implementation strongly rely on (problem dependent) convergence rates of the output quantity of interest over the hierarchy of meshes and the corresponding rate of cost increase to dictate the number of levels and the number of realizations per level. For many engineering problems such parameters are generally estimated through a computational expensive screeening procedure performed before the actual uncertainty analysis. In a previous work we presented a robust and efficient Continuation Multi Level Monte Carlo (C-MLMC) approach, ${ }^{9}$ following the idea of Collier et al. ${ }^{10}$ which is capable of propagating the operational and geometrical uncertainties in compressible inviscid flow problems. The key parameters that control the number of levels and the number of realizations per level are computed on the fly using an online least squares fitting.

In this work we further extend the C-MLMC methodology to compute in one run the expectation and the variance of quantities of interest of the problem under investigation and combine it with a Covariance Matrix Adaptation Evolutionary Strategy (CMA-ES) to perform robust design optimization of airfoils affected by operating and geometric uncertainties.

The paper is organized as follows. Section II introduce the robust design optimization problem and presents how to compute robust loss functions and probabilistic constraints with MLMC. Section III recalls the MLMC method and explain how to calibrate the C-MLMC to compute expectations and variances of a quantity of interest. In Section IV the implementation of the approach is presented. Section V presents the application of the methodology to single and multi objective robust design and finally Section VI concludes the paper and proposes future work. 


\section{Robust Design Optimization with MLMC}

In this work we consider constrained single and multi objective robust design optimization problems. For simplicity, introduce the following notation:

A general formulation of a single objective robust design optimization problem (SO-RDO) reads as follows:

$$
\text { SO-RDO : }\left\{\begin{array}{rl}
\min _{x \in X} & \mathcal{R}\left[\mathbf{Q}_{r}^{q}(\widetilde{x}(x, \omega), p(\psi))\right] \\
\text { s.t } \mathcal{C}_{i}\left[\mathbf{Q}_{c}^{m}(\widetilde{x}(x, \omega), p(\psi))\right] \leq k_{i} \quad i=1, \ldots, s \\
\quad x_{L} \leq x \leq x_{U}
\end{array}\right.
$$

where

$$
\mathbf{Q}_{r}^{q}(\widetilde{x}(x, \omega), p(\psi))=\left[Q_{r_{1}}(\widetilde{x}(x, \omega), p(\psi)), \ldots, Q_{r_{q}}(\widetilde{x}(x, \omega), p(\psi))\right]
$$

is the vector of dimension $q$ of QoI that have to be robustified and

$$
\mathbf{Q}_{c}^{m}(\widetilde{x}(x, \omega), p(\psi))=\left[Q_{c_{1}}(\widetilde{x}(x, \omega), p(\psi)), \ldots, Q_{c_{m}}(\widetilde{x}(x, \omega), p(\psi))\right]
$$

the vector (of dimension $m$ ) of QoI that have to be constrained. $x$ is the vector of design variable. Its actual realization $\widetilde{x}(x, \omega)$ as well as the vector of system parameters $p(\psi)$ may be affected by uncertainties. The design variable vector belong to the subset $X$ of $\mathbb{R}^{n}$ with $n$ being the number of design variables. We denote the uncertainties by $\omega \in \Omega$ and $\psi \in \Psi$, where $\Omega$ and $\Psi$ are respectively the uncertainty spaces of the design and system variables. $\mathcal{R}$ is the robust loss function (or fitness in case of maximization) involving one or more QoI $Q_{r}$ (e.g. weighted sum) that have to be optimized and robustified. $\mathcal{C}_{i}$ denotes a set of deterministic and probabilistic constraints ( $s$ is the number of constraints) applied on a set of QoI $\mathbf{Q}_{c}^{m} \cdot x_{L}$ and $x_{U}$ are lower and upper bounds of the design variable.

Definition 1. The feasible design space $\widetilde{X}$ is defined as the set $\left\{x \mid \mathcal{C}_{i}\left[\mathbf{Q}_{c}^{m}(\widetilde{x}(x, \omega), p(\psi))\right] \leq k_{i}, i=1, \ldots, s\right.$ and $\left.x_{L} \leq x \leq x_{U}\right\}$

The loss function $\mathcal{R}$ is a measure of robustness against the uncertainties in the design and system parameters. Classical robust optimization approaches generally consider optimizing performance under 'worst-case' outcomes (min-max formulations). This approach is known to generally produce overly conservative designs with suboptimal performance in the uncertainty range.

Hereafter we consider loss functions that involve the weighted sum of the mean $\mu_{Q_{r}}$ and standard deviation $\sigma_{Q_{r}}$ of the quantity of interest that have to be optimized:

$$
\mathcal{R}\left[Q_{r}(\widetilde{x}(x, \omega), p(\psi))\right]=w_{1} \mu_{Q_{r}}[\widetilde{x}(x, \omega), p(\psi)]+w_{2} \sigma_{Q_{r}}[\widetilde{x}(x, \omega), p(\psi)]
$$

$w_{1}$ and $w_{2}$ denote respectively the weights for the mean and standard deviation of $Q_{r}$. It is worth underlining that the present approach can also be extended to include higher statistical moments, measures of asymmetry or tailedness of the probability density function (skewness and kurtosis), quantiles, conditional value at risk (CVaR) or more complex coherent risk measures.

The constraint function $\mathcal{C}$ involves also a weighted sum of deterministic values and statistical moments.

$$
\mathcal{C}\left[Q_{c}(\widetilde{x}(x, \omega), p(\psi))\right]=g_{0}+g_{1} \mu_{Q_{c}}[\widetilde{x}(x, \omega), p(\psi)]+g_{2} \sigma_{Q_{c}}[\widetilde{x}(x, \omega), p(\psi)]
$$

where $g_{0}, g_{1}$ and $g_{2}$ are the weights of the sum.

We now define with $\mathbf{R}^{\Phi}\left[\mathbf{Q}_{r}^{q}(\widetilde{x}(x, \omega), p(\psi))\right]$ a vector of $\Phi$ robust loss functions:

$$
\mathbf{R}^{\Phi}\left[\mathbf{Q}_{r}^{q}(\widetilde{x}(x, \omega), p(\psi))\right]=\left[\mathcal{R}_{1}\left[\mathbf{Q}_{r}^{q}(\widetilde{x}(x, \omega), p(\psi))\right], \ldots, \mathcal{R}_{\Phi}\left[\mathbf{Q}_{r}^{q}(\widetilde{x}(x, \omega), p(\psi))\right]\right]
$$

Using the same notation we can further generalize (1) and define the multi-objective robust design optimization problem (MO-RDO) as :

$$
\text { MO-RDO : }\left\{\begin{aligned}
\underset{x \in X}{\mathrm{P}-\min } & \mathbf{R}^{\Phi}\left[\mathbf{Q}_{r}^{q}(\widetilde{x}(x, \omega), p(\psi))\right] \\
\text { s.t } & \mathcal{C}_{i}\left[\mathbf{Q}_{c}^{m}(\widetilde{x}(x, \omega), p(\psi))\right] \leq k_{i} \quad i=1, \ldots, s \\
& x_{L} \leq x \leq x_{U}
\end{aligned}\right.
$$

where $\Phi$ is the number of objective functions and $P-\min _{x \in X} \quad \mathbf{R}^{\Phi}$ denote all Pareto optimal values of $\mathbf{R}^{\Phi}$. 
Definition 2. A feasible point $x^{*} \in \widetilde{X}$ is Pareto optimal if and only if there does not exist another feasible point $x \in \widetilde{X}$ such that $\boldsymbol{R}^{\Phi}\left[\mathbf{Q}_{r}^{q}(\widetilde{x}(x, \omega), p(\psi))\right] \leq \boldsymbol{R}^{\Phi}\left[\mathbf{Q}_{r}^{q}\left(\widetilde{x}\left(x^{*}, \omega\right), p(\psi)\right)\right]$, and $\mathcal{R}_{i}\left[\mathbf{Q}_{r}^{q}(\widetilde{x}(x, \omega), p(\psi))\right]<$ $\mathcal{R}_{i}\left[\mathbf{Q}_{r}^{q}\left(\widetilde{x}\left(x^{*}, \omega\right), p(\psi)\right)\right](i=1, \ldots, \Phi)$ for at least one objective.

In the previous definition the relation $\mathbf{a} \leq \mathbf{b}$ between vectors has to be interpreted component-wise.

The main difference between SO-RDO and MO-RDO is that in the latter usually there is no single solution, but a set of points that fit a predetermined definition for optimum.

In this work we employ Single and Multi Objective Covariance Matrix Adaptation Evolutionary Strategies (CMA-ES) to solve the SO-RDO (1) and MO-RDO (7) problems. CMA-ES are a class of stochastic derivativefree evolutionary algorithms for numerical optimization of non-linear and non-convex black-box optimization problems introduced by Hansen ${ }^{11,12}$ that estimate a covariance matrix associated to the current population within an iterative procedure. New individuals (candidate solutions) are sampled at each generation (iteration of the algorithm) according to a multivariate normal distribution in $\mathbb{R}^{n}$ with such covariance matrix. The CMA-ES does not use gradient informations and does not even assume their existence. This makes the method feasible on non-smooth and non-continuous problems.

The novelty and the main contribution of this work is in the methodology employed to compute the robust loss function $\mathcal{R}$ and the set of probabilistic constraints $\mathcal{C}$. We developed a Continuation Multi Level Monte Carlo (C-MLMC) algorithm capable of efficiently computing statistics of all the required QoI. The appropriate number of levels and simulations per level is determined on the fly using Bayesian updates. By combining the CMA-ES and our implementation of C-MLMC we are able to optimally control the cost required to compute each individuals loss function, up to a prescribed tolerance, in the population of candidate solutions and guarantee a prescribed tolerance on the statistics of the QoIs.

\section{II.A. Computation of $\mathcal{R}$ and $\mathcal{C}$ by MLMC}

For the computation of $\mathcal{R}$ and $\mathcal{C}$ we are interested in the expected value $\mu_{Q}=\mathbb{E}[Q]$ and variance $\sigma_{Q}^{2}=\operatorname{Var}[Q]$ of a QoI $Q$ associated to the solution $u$ of an aerodynamic problem computed using a numerical approximation with a discretization parameter $M$ (number of spatial degrees of freedom). $Q$ is approximated by $Q_{M}=f\left(u_{M}\right)$. The key idea of $\mathrm{MLMC}^{8}$ is to simultaneously draw Monte Carlo (MC) samples on several approximations $Q_{M_{l}}$ of $Q$ build on a hierarchy of computational grids, called levels, with discretization parameters $M_{0}<$ $M_{1}<\ldots<M_{L}=M$. The linearity of the expectation operator suggests that the expectation of the QoI on the finest level can be written as a telescopic sum of the expectation of the QoI on the coarsest level plus a sum of correction terms adding the difference in expectation between evaluations on consecutive levels:

$$
\mathbb{E}\left[Q_{M_{L}}\right]=\mathbb{E}\left[Q_{M_{0}}\right]+\sum_{l=1}^{L} \mathbb{E}\left[Q_{M_{l}}-Q_{M_{l-1}}\right]=\sum_{l=0}^{L} \mathbb{E}\left[Y_{l}\right]
$$

where the correction terms $Y_{l}=Q_{M_{l}}-Q_{M_{l-1}}$ are computed using the same sample on both levels whereas corrections on different levels are sampled independently.

The Multi Level Monte Carlo estimator for $\mathbb{E}[Q]$ is:

$$
\mathrm{E}^{\mathrm{MLMC}}\left[Q_{M}\right]:=\sum_{l=0}^{L} \mathrm{E}^{\mathrm{MC}}\left[Q_{M_{l}}-Q_{M_{l-1}}\right]=\sum_{l=0}^{L} \mathrm{E}^{\mathrm{MC}}\left[Q_{M_{l}}\right]-\mathrm{E}^{\mathrm{MC}}\left[Q_{M_{l-1}}\right]
$$

where $\mathrm{E}^{\mathrm{MC}}\left[Q_{M_{l}}\right]$ and $\mathrm{E}^{\mathrm{MC}}\left[Q_{M_{l-1}}\right]$ are the MC expectation estimators for $\mathbb{E}\left[Q_{M}\right]$ computed on level $l$ and $l-1$ :

$$
\mathrm{E}^{\mathrm{MC}}\left[Q_{M}\right]:=\frac{1}{N} \sum_{i=1}^{N} Q_{M}\left(\omega^{(i)}\right)
$$

The Multi Level Monte Carlo (MLMC) estimator for $\operatorname{Var}[Q]$ can be easily constructed following the same approach as:

$$
\mathrm{V}^{\mathrm{MLMC}}\left[Q_{M}\right]:=\sum_{l=0}^{L} \mathrm{~V}^{\mathrm{MC}}\left[Q_{M_{l}}\right]-\mathrm{V}^{\mathrm{MC}}\left[Q_{M_{l-1}}\right]
$$


where $\mathrm{V}^{\mathrm{MC}}\left[Q_{M_{l}}\right]$ and $\mathrm{V}^{\mathrm{MC}}\left[Q_{M_{l-1}}\right]$ are the $\mathrm{MC}$ variance estimators for $\operatorname{Var}\left[Q_{M}\right]$ computed on level $l$ and $l-1$ :

$$
\mathrm{V}^{\mathrm{MC}}\left[Q_{M}\right]:=\frac{1}{N-1} \sum_{i=1}^{N}\left(Q_{M}\left(\omega^{(i)}\right)-\mathrm{E}^{\mathrm{MC}}\left[Q_{M}\right]\right)^{2} .
$$

In a previous work ${ }^{13}$ we presented an efficient MLMC algorithm to propagate the operating and geometric uncertainties in internal and external aerodynamic problem. To overcome some of the limitations of classical MLMC approach and to further reduce the computational burden required to accurately compute the problem dependent parameters needed to estimate the number of level and samples per levels required to achieve a prescribed tolerance we recently developed a continuation algorithm following the idea of Collier et al. ${ }^{10}$

As underlined and extensively studied in Pisaroni et al. ${ }^{9}$ the common practice of computing the number of levels and simulations per levels by relying on rates computed a-priori using a screening procedure can be quite computationally expensive. Additionally if the number of screening levels and samples per level are chosen too small, the extrapolation of the convergence rates might be quite unstable, leading to an unreliable estimation or an over conservative (more computational demanding) simulation. We assessed the efficiency and dimension independent (number of uncertain parameters) convergence rates of the C-MLMC approach on compressible inviscid aerodynamics problems ${ }^{9}$ and recently on viscous problems modeled by RANS equations. Thanks to the rigorous mean square error estimators that exist for the MLMC estimators for mean and variance we are able to approximate, with different levels of accuracy, in just one run of our C-MLMC algorithm the following quantities:

- mean and standard deviation of the QoI that has to be optimized: $\mu_{Q_{r}}[x(\omega), p(\psi)], \sigma_{Q_{r}}[x(\omega), p(\psi)]$

- mean and standard deviation of the QoI that has to be constrained: $\mu_{Q_{c}}[x(\omega), p(\psi)], \sigma_{Q_{c}}[x(\omega), p(\psi)]$

\section{C-MLMC for $\mathrm{E}^{\mathrm{MLMC}}\left[Q_{M}\right]$ and $\mathrm{V}^{\mathrm{MLMC}}\left[Q_{M}\right]$}

\section{III.A. Calibration of C-MLMC for $\mathrm{E}^{\mathrm{MLMC}}\left[Q_{M}\right]$}

We recall here briefly the procedure to optimally tune the MLMC algorithm in the Continuation version developed in. ${ }^{9,10}$ We assume that the sequence of discretizations (with parameters $M_{0}<M_{1}<\ldots<M_{L}=M$ ) provide errors that decrease algebraically with $M_{l}$, with cost increasing algebraically in $M_{l}$ (see Figure 1). More precisely:

$\widetilde{\mathbf{A}} 1$. Let $c_{\gamma}, \gamma>0$ be such that the cost $C_{l}$ to compute one sample $Q_{M_{l}}$ at level $l$ is:

$$
C_{l}=C\left(Q_{M_{l}}\left(\omega^{(i)}\right)\right) \leq c_{\gamma} M_{l}^{\gamma},
$$

Â2. Let $c_{\alpha}, \alpha>0$ be such that:

$$
\left|\mathbb{E}\left[Q_{M_{l}}-Q\right]\right| \leq c_{\alpha} M_{l}^{-\alpha}
$$

$\widetilde{\mathbf{A}} 3$. Let $c_{\beta}, \beta>0$, with $\alpha \geq \min (\beta, \gamma)$ be such that:

$$
\operatorname{Var}\left[Y_{l}\right] \leq c_{\beta} M_{l}^{-\beta},
$$

These rates $(\alpha, \beta, \gamma)$ and constants $\left(c_{\alpha}, c_{\beta}, c_{\gamma}\right)$ will be estimated on the fly by the algorithm.

We require the total error of the MLMC estimator $\mathrm{E}^{\mathrm{MLMC}}\left[Q_{M}\right]$ achieves the desired tolerance $\varepsilon$ with confidence probability $1-\phi$ (exploiting the asymptotic normality of the estimator $\left.\mathrm{E}^{\mathrm{MLMC}}\left[Q_{M}\right]^{10}\right)$ :

$$
\begin{aligned}
\operatorname{TErr} & :=\left|E^{\mathrm{MLMC}}\left[Q_{M}\right]-\mathbb{E}[Q]\right| \leq\left|\mathbb{E}\left[Q-Q_{M}\right]\right|+\left|\mathrm{E}^{\mathrm{MLMC}}\left[Q_{M}\right]-\mathbb{E}\left[Q_{M}\right]\right| \\
& \leq \underbrace{\left|\mathbb{E}[Q]-\mathbb{E}\left[Q_{M}\right]\right|}_{\mathbf{B}}+\mathcal{C}_{\phi} \sqrt{\underbrace{\operatorname{Var}\left[\mathrm{E}^{\mathrm{MLMC}}\left[Q_{M}\right]\right]}_{\text {SE }}}
\end{aligned}
$$


where $\mathcal{C}_{\phi}=\Phi^{-1}\left(1-\frac{\phi}{2}\right)$ and $\Phi$ is the cumulative distribution function (CDF) of a standard normal random variable. We introduce a splitting parameter $\theta \in(0,1)$ and require in our simulations that:

$$
\begin{gathered}
\text { Bias: } \quad \mathbf{B}:=\left|\mathbb{E}[Q]-\mathbb{E}\left[Q_{M}\right]\right| \leq(1-\theta) \varepsilon \\
\text { Statistical Error: } \quad \mathbf{S E}:=\operatorname{Var}\left[\mathrm{E}^{\mathrm{MLMC}}\left[Q_{M}\right]\right]=\sum_{l=0}^{L} \frac{\operatorname{Var}\left[Y_{l}\right]}{N_{l}} \leq\left(\frac{\theta \varepsilon}{\mathcal{C}_{\phi}}\right)^{2}
\end{gathered}
$$

The bias contribution $\mathbf{B}$, in absence of an exact solution $\mathbb{E}[Q]$ of the problem under consideration, is approximated as:

$$
\mathbf{B} \approx\left|\mathrm{E}^{\mathrm{MC}}\left[Q_{L}-Q_{L-1}\right]\right|
$$

that is a reasonable estimate of the discretization error for sufficiently fine grids.

Instead of solving the MLMC hierarchy for the final tolerance $\varepsilon$ (as in the classical MLMC approach), we solve for the QoI with a sequence of decreasing tolerances and progressively improve the estimation of the problem dependent parameters that directly control the number of levels and sample sizes. To achieve a certain RMSE of $\varepsilon$, we prescribe a slightly smaller tolerance $\frac{\varepsilon}{r_{2}}$ with $r_{2}>1$ and define a sequence of decreasing tolerances $\varepsilon_{0}>\varepsilon_{1}>\cdots>\varepsilon_{i}>\cdots>\varepsilon_{k}=\frac{\varepsilon}{r_{2}}$ with $\varepsilon_{i}=r_{1} \varepsilon_{i+1}$ and $r_{1}>1$ where, for a given $\varepsilon_{0}>\varepsilon$, the number $\mathcal{I}_{\varepsilon}$ of iterations is given by:

$$
\mathcal{I}_{\varepsilon}=\left\lfloor\frac{-\log \left(\frac{\varepsilon}{r_{2}}\right)+\log \left(\varepsilon_{0}\right)}{\log \left(r_{1}\right)}\right\rfloor .
$$

At the $i$-th iteration of the C-MLMC algorithm (with prescribed tolerance $\varepsilon_{i}$ ), the optimal number of levels is computed by solving the following discrete optimization problem and by exhaustive search:

$$
\left(L_{i}, \theta_{i}\right)=\underset{\substack{L \in\left[L_{i-1}, \ldots, L_{M A X}\right], \theta \in(0,1) \\ \text { s.t. } c_{\alpha} M_{L}^{-\alpha}=(1-\theta) \varepsilon_{i}}}{\arg \min } \mathbf{C}_{\mathrm{MLMC}}\left(\varepsilon_{i}, \theta, L\right)
$$

using the cost model:

$$
\mathbf{C}_{\mathrm{MLMC}}\left(\varepsilon_{i}, \theta, L\right)=\left(\frac{\mathcal{C}_{\phi}}{\theta \varepsilon_{i}}\right)^{2}\left(\sum_{l=0}^{L} \sqrt{C_{l} \mathbb{V} a r\left[Y_{l}\right]}\right)^{2}
$$

obtained with an optimal choice of $N_{l}$ computed with:

$$
N_{l}=\left\lceil\left(\frac{\mathcal{C}_{\phi}}{\theta \varepsilon}\right)^{2} \sqrt{\frac{\mathbb{V a r}\left[Y_{l}\right]}{C_{l}}} \sum_{k=0}^{L} \sqrt{C_{k} \operatorname{Var}\left[Y_{k}\right]}\right\rceil \quad l=0,1, \ldots, L .
$$

The constraint $c_{\alpha} M_{L}^{-\alpha}=(1-\theta) \varepsilon_{i}$ in (20) represents the bias constrain and allows to determine $\theta$ as a function of $L$ (and $\left.\varepsilon_{i}\right)$ :

$$
\theta\left(\varepsilon_{i}, L\right)=1-\frac{c_{\alpha} M_{L}^{-\alpha}}{\varepsilon_{i}}
$$

Since $c_{\alpha} M_{L}^{-\alpha}$ can take only discrete values, for each $L$ that satisfies the bias constraint $\mathbf{B} \leq \varepsilon_{i}$, it is worth taking the largest possible $\theta=1-\frac{\mathbf{B}}{\varepsilon_{i}}$ so as to relax as much as possible the statistical error constraint

$$
\operatorname{Var}\left[\mathrm{E}^{\mathrm{MLMC}}\left[Q_{M}\right]\right] \leq\left(\frac{\theta \varepsilon_{i}}{C_{\phi}}\right)^{2}
$$

and reduce the overall computational cost.

In the classical MLMC approach $\mathbb{V} a r\left[Y_{l}\right]$ in (22) and (21) is approximated using the MC level sample variance $\mathrm{V}^{\mathrm{MC}}\left[Y_{l}\right]$ :

$$
\operatorname{Var}\left[Y_{l}\right] \approx \mathrm{V}^{\mathrm{MC}}\left[Y_{l}\right]=\frac{1}{N_{l}-1} \sum_{n=1}^{N_{l}}\left(Y_{l}\left(\omega^{(n, l)}\right)-\mathrm{E}^{\mathrm{MC}}\left[Y_{l}\right]\right)^{2}
$$

In the C-MLMC approach the estimation of the variances $\operatorname{Var}\left[Y_{l}\right]$ is performed using a Bayesian methodology that uses samples generated on all levels to locally improve the estimation. Using the bias model $\mathbb{E}\left[Y_{l}\right] \approx$ 
$\widehat{\mu}_{l}:=c_{\alpha} M_{l}^{-\alpha}$ and variance model $\operatorname{Var}\left[Y_{l}\right] \approx \widehat{\lambda}_{l}^{-1}:=c_{\beta} M_{l}^{-\beta}$ with $c_{\alpha}, \alpha, c_{\beta}, \beta$ estimated from the previous iteration of the CMLMC algorithm, $Y_{l}$ is described as a Gaussian random variable $\mathcal{N}\left(\mu_{l}, \lambda_{l}^{-1}\right)$. The Bayesian update of $\mu_{l}$ and $\lambda_{l}^{-1}$ is performed based on the collected values $Y_{l}\left(\omega^{(n, l)}\right)$ with a Normal-Gamma prior distribution $\mathcal{N G}\left(\widehat{\lambda}_{l}, k_{0}, k_{1} \widehat{\lambda}_{l}+1 / 2, k_{1}\right)$ with maximum in $\left(\widehat{\mu}_{l}, \widehat{\lambda}_{l}\right)\left(k_{0}\right.$ and $k_{1}$ are two parameters that represent our "certainty" on $\widehat{\mu}_{l}$ and $\left.\widehat{\lambda}_{l}^{-1}\right)$. The posterior is also a Normal-Gamma, with maximum at

$$
\mu_{l}^{M A P}=\frac{N_{l} \mathrm{E}^{\mathrm{MC}}\left[Y_{l}\right]+k_{0} \widehat{\mu}_{l}}{k_{0}+N_{l}} \quad \text { and } \quad \lambda_{l}^{M A P}=\frac{\Xi_{1, l}-\frac{1}{2}}{\Xi_{2, l}}
$$

with:

$$
\begin{aligned}
& \Xi_{1, l}=\frac{1}{2}+\widetilde{k_{1}} \widehat{\lambda}_{l}+\frac{N_{l}}{2}, \\
& \Xi_{2, l}=\widetilde{k_{1}}+\frac{N_{l}-1}{2} \mathrm{~V}^{\mathrm{MC}}\left[Y_{l}\right]+\frac{k_{0} N_{l}\left(\mathrm{E}^{\mathrm{MC}}\left[Y_{l}\right]-\widehat{\mu}_{l}\right)^{2}}{2\left(k_{0}+N_{l}\right)} .
\end{aligned}
$$

The resulting update formula for $\operatorname{Var}\left[Y_{l}\right] \approx \lambda_{l}^{-1}$ is then:

$$
\mathrm{V}^{\mathrm{C}}\left[Y_{l}\right]:=\frac{\Xi_{2, l}}{\Xi_{1, l}-\frac{1}{2}} \quad l>0
$$

Following the above arguments, $\mathrm{V}^{\mathrm{C}}\left[Y_{l}\right]$ is then used to in (21) and (22) to approximate $\operatorname{Var}\left[Y_{l}\right]$. On the other hand, the sample variance 25 is used to estimate the final statistical error SE $17 \mathrm{~b}$.
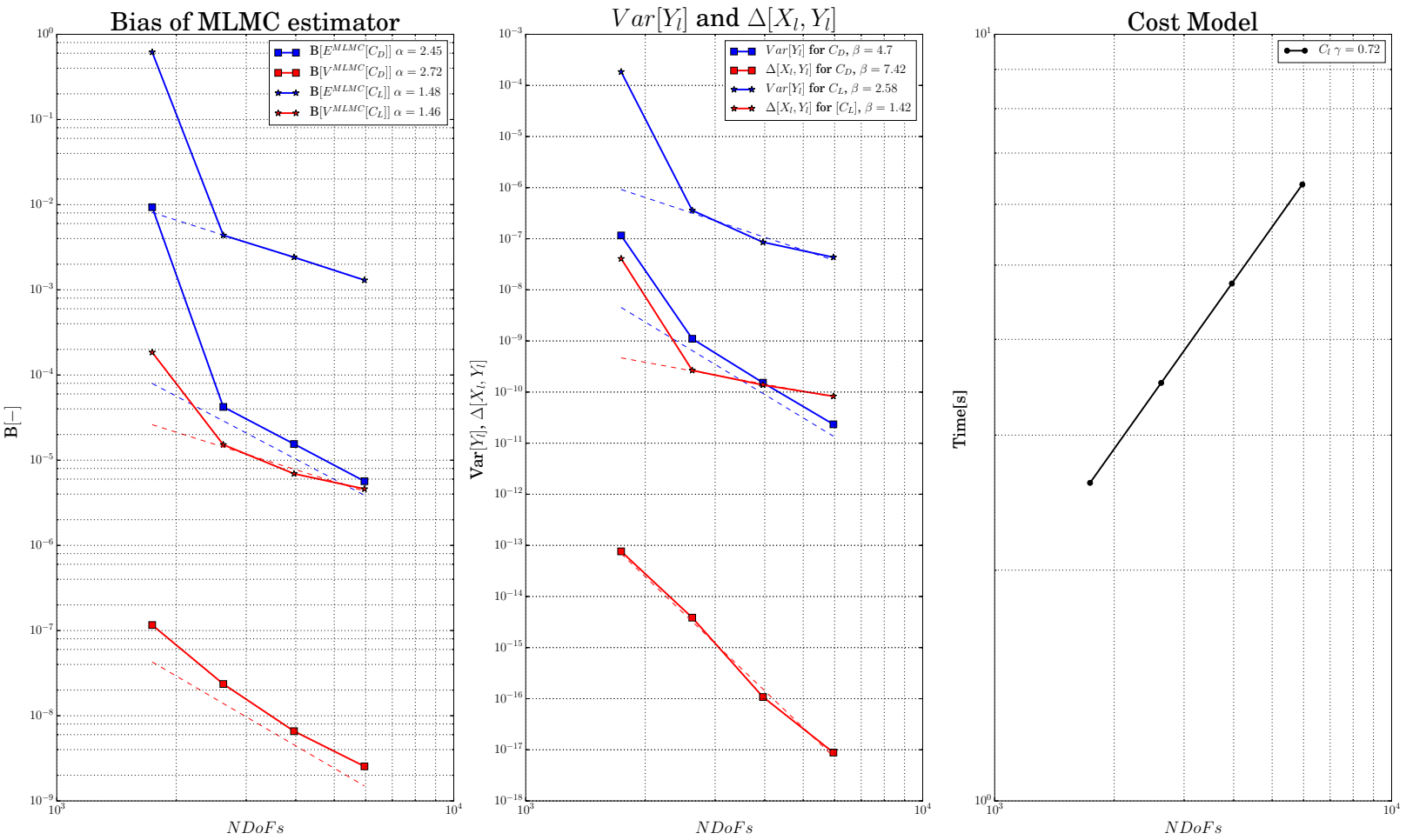

Figure 1: Bias of the MLMC estimators $\mathrm{E}^{\mathrm{MLMC}}\left[Q_{M}\right]$ and $\mathrm{V}^{\mathrm{MLMC}}\left[Q_{M}\right], \operatorname{Var}\left[Y_{l}\right]$ and $\Delta\left[X_{l}, Y_{l}\right]$ decays and cost model. The QoI are the $C_{D}$ and $C_{L}$ coefficients for a UQ analysis of the RAE-2822 in transonic regime. The $\mathrm{y}$-axis in the first two plots is the absolute error, while the $\mathrm{x}$-axis denotes the number of degrees of freedom of the level grids. 


\section{III.B. Calibration of C-MLMC for $\mathrm{V}^{\mathrm{MLMC}}\left[Q_{M}\right]$}

Following the same arguments presented above for $\mathrm{E}^{\mathrm{MLMC}}\left[Q_{M}\right]$, we now apply the above presented C-MLMC procedure to $\mathrm{V}^{\mathrm{MLMC}}\left[Q_{M}\right]$. The only difference is in the requirements of the total error of the MLMC estimator. We require that $\mathrm{V}^{\text {MLMC }}\left[Q_{M}\right]$ achieves the desired tolerance $\varepsilon$ with confidence probability $1-\phi$ :

$$
\begin{aligned}
\operatorname{TErr} & :=\left|\mathrm{V}^{\mathrm{MLMC}}\left[Q_{M}\right]-\operatorname{Var}[Q]\right| \leq\left|\operatorname{Var}[Q]-\operatorname{Var}\left[Q_{M}\right]\right|+\left|\mathrm{V}^{\mathrm{MLMC}}\left[Q_{M}\right]-\operatorname{Var}\left[Q_{M}\right]\right| \\
& \leq \underbrace{\left|\operatorname{Var}[Q]-\operatorname{Var}\left[Q_{M}\right]\right|}_{\mathbf{B}}+\mathcal{C}_{\phi} \sqrt{\underbrace{\operatorname{Var}\left[\mathrm{V}^{\mathrm{MLMC}}\left[Q_{M}\right]\right]}_{\text {SE }}}
\end{aligned}
$$

We introduce again a splitting parameter $\theta \in(0,1)$ and require in our simulations (see Bierig et al. ${ }^{14}$ ) that:

$$
\begin{aligned}
& \mathbf{B}:=\left|\operatorname{Var}[Q]-\operatorname{Var}\left[Q_{M}\right]\right| \leq(1-\theta) \varepsilon, \\
& \mathbf{S E}:=\operatorname{Var}\left[\mathrm{V}^{\mathrm{MLMC}}\left[Q_{M}\right]\right]=\sum_{l=0}^{L} \mathbb{E}\left[\left(\mathrm{C}^{\mathrm{MC}}\left[X_{l}, Y_{l}\right]-\mathbb{C}\left[X_{l}, Y_{l}\right]\right)^{2}\right]=\sum_{l=0}^{L} \frac{1}{N_{l}} \boldsymbol{\Delta}\left[X_{l}, Y_{l}\right]
\end{aligned}
$$

with $X_{l}=Q_{M_{l}}+Q_{M_{l-1}}$ and $Y_{l}=Q_{M_{l}}-Q_{M_{l-1}}, \mathbb{C}\left[X_{l}, Y_{l}\right]$ denotes the covariance of $X_{l}$ and $Y_{l}$ :

$$
\mathbb{C}\left[X_{l}, Y_{l}\right]=\mathbb{E}\left[\left(X_{l}-\mathbb{E}\left[X_{l}\right]\right)\left(Y_{l}-\mathbb{E}\left[Y_{l}\right]\right)\right] .
$$

and $\mathrm{C}^{\mathrm{MC}}\left[X_{l}, Y_{l}\right]$ the MC covariance estimator of $\mathbb{C}\left[X_{l}, Y_{l}\right]$ :

$$
\mathrm{C}^{\mathrm{MC}}\left[X_{l}, Y_{l}\right]=\frac{1}{N_{l}-1} \sum_{i=1}^{N_{l}}\left(X_{l}\left(\omega^{(i)}\right)-\mathrm{E}^{\mathrm{MC}}\left[X_{l}\right]\right)\left(Y_{l}\left(\omega^{(i)}\right)-\mathrm{E}^{\mathrm{MC}}\left[Y_{l}\right]\right)
$$

and

$$
\boldsymbol{\Delta}\left[X_{l}, Y_{l}\right]=N_{l} \mathbb{E}\left[\left(\mathrm{C}^{\mathrm{MC}}\left[X_{l}, Y_{l}\right]-\mathbb{C}\left[X_{l}, Y_{l}\right]\right)^{2}\right]
$$

As it is possible to see from the previous error contributions, the key difference between $\mathrm{E}^{\mathrm{MLMC}}\left[Q_{M}\right]$ and $\mathrm{V}^{\mathrm{MLMC}}\left[Q_{M}\right]$ is in the treatment of the bias $\mathbf{B}$ and statistical error contributions SE.

It is possible to show (see Appendix A) that:

$$
\boldsymbol{\Delta}\left[X_{l}, Y_{l}\right]=\left(\mathbb{E}\left[X_{l}^{2} Y_{l}^{2}\right]-\frac{N-2}{N-1} \mathbb{E}\left[X_{l} Y_{l}\right]^{2}+\frac{1}{N-1} \operatorname{Var}\left[X_{l}\right] \operatorname{Var}\left[Y_{l}\right]\right)
$$

We can write the statistical error for $\operatorname{V~}^{\mathrm{MLMC}}\left[Q_{M}\right]$ as:

$$
\operatorname{Var}\left[\mathrm{V}^{\mathrm{MLMC}}\left[Q_{M}\right]\right]=\sum_{l=0}^{L} \frac{1}{N_{l}}\left(\mathbb{E}\left[X_{l}^{2} Y_{l}^{2}\right]-\frac{N-2}{N-1} \mathbb{E}\left[X_{l} Y_{l}\right]^{2}+\frac{1}{N-1} \operatorname{Var}\left[X_{l}\right] \operatorname{Var}\left[Y_{l}\right]\right)
$$

Now, in order to use the C-MLMC methodology presented before for the MLMC expectation estimator $\mathrm{E}^{\mathrm{MLMC}}\left[Q_{M}\right]$ we will simply perform the Bayesian update on $\boldsymbol{\Delta}\left[X_{l}, Y_{l}\right]$ instead of $\operatorname{Var}\left[Y_{l}\right]$.

A crucial task in C-MLMC simulations, that require the solution of a large number of aerodynamic problems, is the efficient and robust computation of bias and statistical error contributions (e.g. in (35)) and performing each deterministic simulation on different levels in parallel.

Naïve one-pass approaches for the computation of statistical moments that involve sum of powers are known to lead to numerical instabilities as well as to arithmetic overflow when dealing with large values. On the other hand, two-pass algorithms (first compute the mean and than the required powers of deviations) are impractical for large data sets since they require the storage of a large number of informations in the cache memory as each data point must be accessed twice. For these reasons numerically stable online one-pass algorithms for centered statistical moments have long been investigated ${ }^{16} .{ }^{17}$ A comprehensive review of parallel online one-pass algorithms for arbitrary order statistical moment and covariances can be found in. ${ }^{18}$ In Appendix B we present a novel one-pass update formula for parallel computation of arbitrary order 'PQ-covariances' $\mathbb{C}_{p q}[X, Y]$. PQ-covariances are special cases of central cross-moments with only two entries:

$$
\left.\mathbb{C}_{p q}[X, Y]=\mathbb{E}\left[\left(X_{l}^{\prime}\right)^{p}\left(Y_{l}^{\prime}\right)^{q}\right]\right]=\mathbb{E}\left[\left(X_{l}-\mathbb{E}\left[X_{l}\right]\right)^{p}\left(Y_{l}-\mathbb{E}\left[Y_{l}\right]\right)^{q}\right], \quad p, q \in \mathbb{N}
$$

Such PQ-covariances can be used to update the terms that appear in the bias and statistical error contributions, the MC estimators $\mathrm{E}^{\mathrm{MC}}$ and $\mathrm{V}^{\mathrm{MC}}$ that appear in the MLMC estimators in (9) and (11) at each iteration of the C-MLMC algorithm and when the simulations are performed in parallel without any storage of informations in the cache memory as they are updated whenever the results of simulations are available. 


\section{Implementation of the Methodology}

In order to solve the single (1) and multi objective (7) robust design optimization problems presented in Section II we adapt the CMA-ES algorithm (see Hansen ${ }^{19}$ for the single objective case and Igel et al. ${ }^{12}$ for the multi objective case). Instead of solving a deterministic problem on a fine computational grid for each candidate design $x_{k}$ that populates the ES population of $\lambda$ individuals, we perform a C-MLMC simulation with tolerance $\varepsilon$ imposed on the highest statistical moment that is required to compute the robust loss function $\mathcal{R}$ and the probabilistic constraint $\mathcal{C}$.

In the following Algorithm 1 we denote with $x_{k}, k=1, \ldots, \lambda$, the candidate designs evaluated at each generation $g$. Let $m, \sigma_{p}$ and $C$ be respectively the mean, standard deviation and correlation matrix of the multivariate normal distribution $\mathcal{N}\left(m, \sigma_{p}^{2} C\right)$ used to sample the candidates $x_{k}$. $\psi$ denotes a subset of $\lambda$ that includes the best candidates. Finally $\Theta$ is a vector of size $g_{M A X}$ (maximum number of generations) that defines how the tolerance of the C-MLMC algorithm should be reduced during the optimization loop.

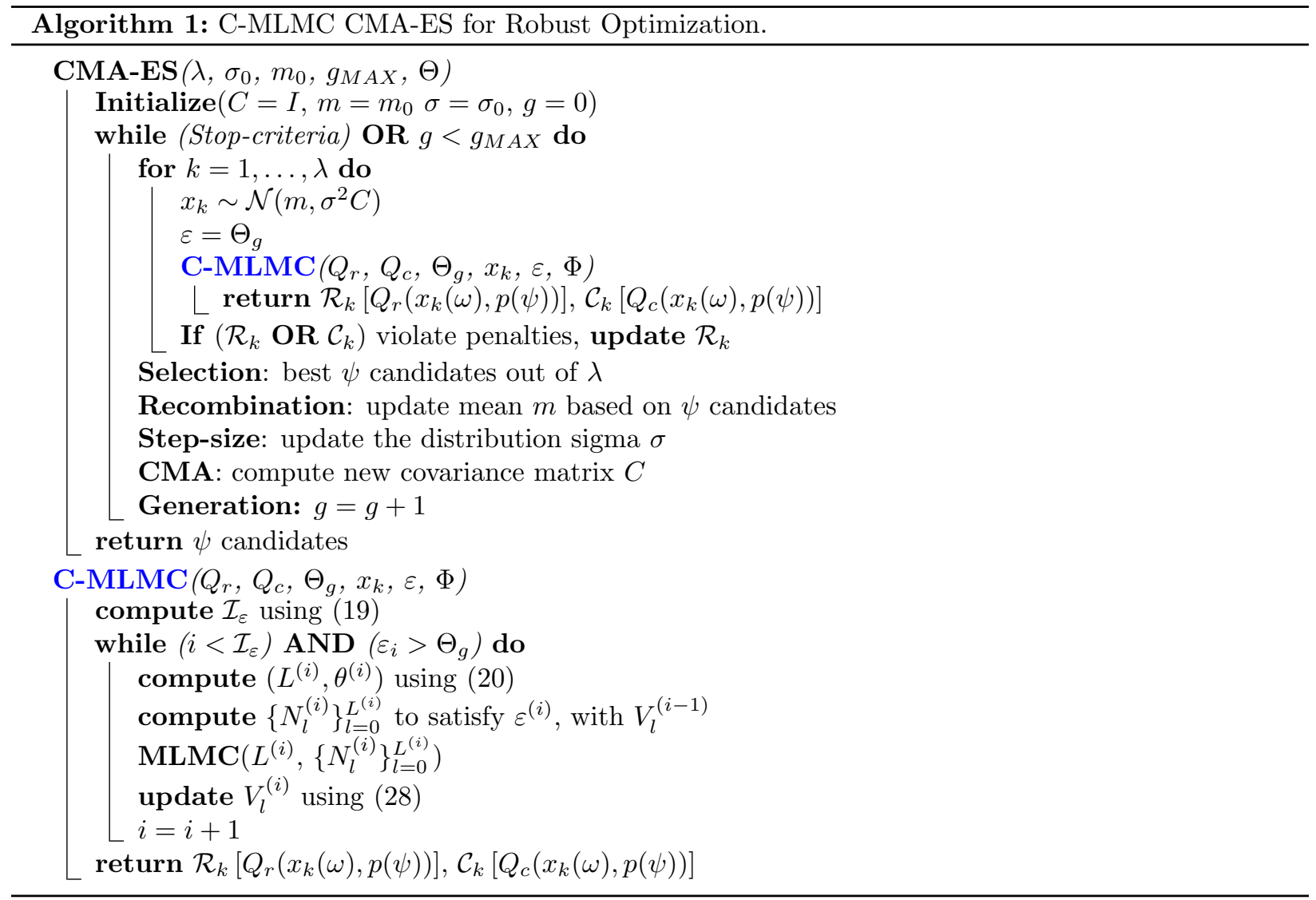

\section{Application to Single and Multi Objective Robust Design Optimization}

In this section, the above presented methodology is applied to single and multi objective robust design optimization of the RAE-2822 airfoil. The design problem involves determining the geometry of an airfoil in order to minimize the drag coefficient $C_{D}$ or maximize lift-drag ratio $L / D$ and their dispersion in an uncertain environment.

C-MLMC Grid Hierarchy and Deterministic Solver. In this work we employ a 4-levels structured grid hierarchy for the C-MLMC simulations. The features of the grid levels are presented in Table 1 along with the average computational time required to compute one deterministic simulation (on one CPU) using the MSES collection of programs for the analysis of airfoils. ${ }^{20}$ MSES solves the steady Euler equations with a finite volume discretization over a streamline grid and is coupled, via the displacement thickness, with a two-equation integral solver for the viscous regions of the boundary layer and trailing wakes. 


\begin{tabular}{|c|c|c|c|}
\hline LEVEL & Airfoil nodes & Cells & CTime $[s]$ \\
\hline$L 0$ & 47 & 1739 & 1.9 \\
\hline$L 1$ & 71 & 2627 & 3.2 \\
\hline$L 2$ & 107 & 3959 & 5.7 \\
\hline$L 3$ & 161 & 5957 & 7.5 \\
\hline$L 4$ & 243 & 8991 & 14.7 \\
\hline
\end{tabular}

Table 1: MLMC 5-levels grid hierarchy for the RAE2822 problem.

Operating Conditions and Uncertainties In the following simulations we consider two uncertain scenarios. First we consider only operating uncertainties (see Table 2) affecting the flow surrounding the airfoil and we use as design variables the Free Form Deformation (FFD) box coefficients (Figure 2(a)).

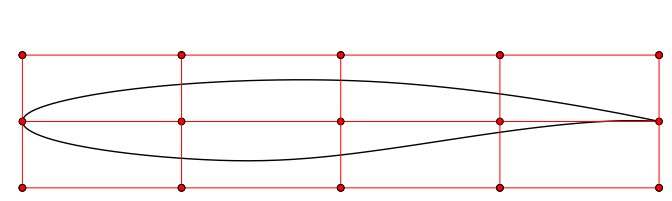

(a) FFD box for RAE2822

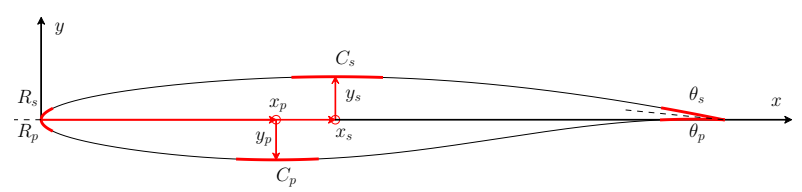

(b) PARSEC parametrization for RAE2822

Figure 2: Preliminary results for MO-RDO2

Another set of simulations is performed considering operating uncertainties and geometric uncertainties due to manufacturing tolerances at the same time. In this scenario the geometrical uncertain set and the design set are the PARSEC coefficients of the airfoil (see Table 3 and Figure 2(b)).

All uncertain parameters are modeled as truncated Gaussian random variables (denoted by $y \sim \mathcal{T} \mathcal{N}(\mu, \sigma, a, b)$ in Table 2 and Table 3 ).

\begin{tabular}{|c|c|c|c|}
\hline & Quantity & Reference $(r)$ & Uncertainty $\mathcal{T N}\left(\mu, \sigma, X_{L O}, X_{U P}\right)$ \\
\hline \hline \multirow{4}{*}{ Operating } & $\alpha_{\infty}$ & 2.31 & $\mathcal{T N}(r, 2 \%,-2 \%,+2 \%)$ \\
parameters & $M_{\infty}$ & 0.730 & $\mathcal{T N}(r, 2 \%,-2 \%,+2 \%)$ \\
& $R e_{c}$ & $6.5 \cdot 10^{6}$ & - \\
& $p_{\infty}[P a]$ & 101325 & - \\
& $T_{\infty}[K]$ & 288.5 & - \\
\hline
\end{tabular}

Table 2: Operating parameters and uncertainties for the RAE2822 problem.

Geometrical Constraints :

In the following optimization problems we require and make sure that the selected airfoils (individuals to be evaluated in the evolutionary strategy) have a shape that allows a prescribed trapezoid to be fit inside them without intersecting. The coordinates of the box vertex are: $v 1=(0.13,0.03), v 2=(0.4,0.05)$, $v 3=(0.4,-0.045), v 4=(0.13,-0.03)$. This is a reasonable requirement to built an aircraft wing with

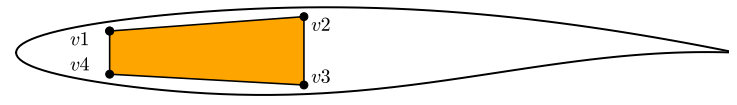
an appropriate inner volume to accommodate the fuel tank and a torque box. Additionally such requirement naturally rejects from the population of candidate individuals all degenerate airfoil shape. Lastly we impose the section of the optimized airfoil to be at least $75 \%$ of the original section of the RAE- 2822 . 


\begin{tabular}{|c|c|c|c|}
\hline & Quantity & PARSEC - RAE-2822 & Uncertainty $\mathcal{T} \mathcal{N}\left(\mu, \sigma, X_{L O}, X_{U P}\right)$ \\
\hline \hline \multirow{3}{*}{ Geometric } & $R_{s}$ & 0.00839 & $\mathcal{T N}(r, 1 \% r,-1 \% r,+1 \% r)$ \\
parameters & $R_{p}$ & 0.00853 & $\mathcal{T N}(r, 1 \% r,-1 \% r,+1 \% r)$ \\
& $x_{s}$ & 0.431 & $\mathcal{T N}(r, 1 \% r,-2 \% r,+2 \% r)$ \\
& $x_{p}$ & 0.346 & $\mathcal{T N}(r, 1 \% r,-2 \% r,+2 \% r)$ \\
& $y_{s}$ & 0.063 & $\mathcal{T N}(r, 1 \% r,-2 \% r,+2 \% r)$ \\
& $y_{p}$ & -0.058 & $\mathcal{T N}(r, 1 \% r,-2 \% r,+2 \% r)$ \\
& $C_{s}$ & -0.432 & $\mathcal{T N}(r, 1 \% r,-1 \% r,+1 \% r)$ \\
& $C_{p}$ & 0.699 & $\mathcal{T N}(r, 1 \% r,-1 \% r,+1 \% r)$ \\
& $\theta_{s}$ & -11.607 & - \\
& $\theta_{p}$ & -2.227 & - \\
\hline
\end{tabular}

Table 3: PARSEC parameters of the RAE2822 airfoil and geometric uncertainties applied on the shape.

\section{V.A. Single Objective Robust Design Optimization - Operating and Geometric Uncertainties}

In this subsection we consider the problem of robustly optimize the shape of the RAE-2822 airfoil affected by operating (system parameters $p(\psi)$, Mach number and angle of attack in Table 2) and geometric uncertainties at the same time (Table 2 and Table 3). The design parameters $x$ are the PARSEC parameters in Table 3 (second column). In the actual stage of the airfoil, uncertainty is added to those parameters (see table 3 last column) We consider two optimization problems. In SO-RDO1, we wish to minimize the drag coefficient of the airfoil and its dispersion while in SO-RDO2, we wish to maximize and robustify the lift-drag ratio.

SO-RDO1 : minimize the sum of the mean and the standard deviation of the drag coefficient $C_{D}$ of the airfoil. We impose a probabilistic constraint on the lift coefficient $C_{L}$ by requiring the mean $\mu_{C_{L}}$ to be greater than or equal to $90 \%$ of the original RAE- $2822\left(C_{L}^{*}=0.9 * C_{L}^{R A E}\right)$. We constrain also the shape of the airfoil by requiring enough space for the fuel/torque box and the final shape to be at least $75 \%$ of the original section of the RAE-2822.

$$
\text { SO-RDO1 : }\left\{\begin{array}{l}
\min _{x} \mathcal{R}\left[C_{D}(\widetilde{x}(x, \omega), p(\psi))\right]=\mu_{C_{D}}(\widetilde{x}(x, \omega), p(\psi))+\sigma_{C_{D}}(\widetilde{x}(x, \omega), p(\psi)) \\
\text { s.t } \mathcal{C}\left[C_{L}(\widetilde{x}(x, \omega), p(\psi))\right]=\mu_{C_{L}}(\widetilde{x}(x, \omega), p(\psi)) \geq C_{L}^{*} \\
\quad x_{L} \leq x \leq x_{U}
\end{array}\right.
$$

In Figure 4 we present the results of SO-RDO1 and compare with the original RAE-2822 and the result of a deterministic optimization of the same airfoil without considering the uncertainties (SO-DO1).

$$
\text { SO-DO1 : }\left\{\begin{array}{l}
\min _{x} C_{D}(x, p) \\
\text { s.t } C_{L}(x, p) \geq C_{L}^{*} \\
\quad x_{L} \leq x \leq x_{U}
\end{array}\right.
$$

As it is possible to observe from Figure 4 the SO-DO1 airfoil is able to achieve the lowest mean drag (circle in the $C_{D}$ plot) and drag at the design condition (Mach $=0.730, \alpha=2.31$, diamond symbol in the $C_{D}$ plot). It is well known that it is generally possible to design an airfoil to have shock-free recompression only for a single combination of Mach-angle of attack. The curvature of the suction inhibits the formation of the shock at design condition (black curve in the $C_{p}$ plot). However as it is possible to observe from the variance of the $C_{p}$, small variations in the geometry of the airfoil and operating conditions, rapidly produce a shock in two regions in the suction side of the airfoil leading to rapid variation in the drag and lift-drag ratio.

On the contrary, the SO-RDO1 airfoil produces a shock on the suction side in all operating conditions also with variations in the geometry of the airfoil. The stability in the intensity and position of the shock is reflected in a small deviation around the mean of the drag coefficient $C_{D}$ and lift drag ratio $L / D$. The relatively large leading edge radius of the robust airfoil and the suction side curvature are able to produce a pressure envelope that reduces progressively the flow before the shock region leading to a weaker shock (compared to the RAE-2822 and SO-DO1). 

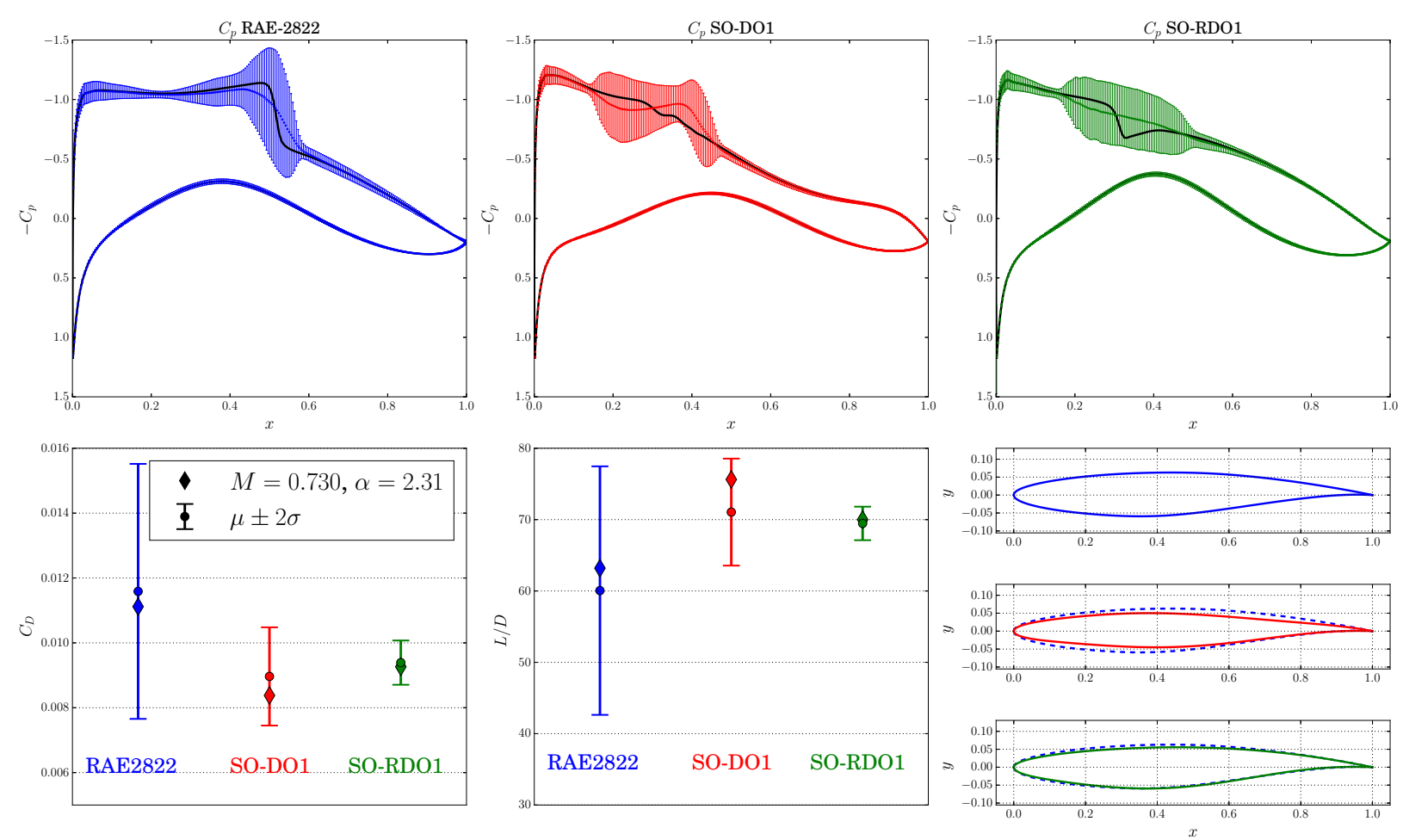

Figure 4: Performances of the SO-RDO1 and SO-DO1 airfoil compared with the original RAE-2822. The upper plots present the uncertain $C_{p}$ profile of the airfoils under operating and geometric uncertainties. The lower plots present the airfoil $C_{D}$ and $L / D$ variations (mean \pm two standard deviations). The diamond symbol represents the performance of the airfoil at design condition (airfoil operating at Mach $=0.730, \alpha=2.31$ ). 
SO-RDO2 : maximise the sum of the mean and the standard deviation of the lift-drag ratio $L / D$ of the airfoil. We constrain again the shape of the airfoil by requiring enough space for the fuel/torque box and the final shape to be at lease $75 \%$ of the original section of the RAE-2822.

$$
\begin{gathered}
\text { SO-RDO2 : }\left\{\begin{aligned}
\max _{x} \mathcal{R}[L / D(\widetilde{x}(x, \omega), p(\psi))]=\mu_{L / D}(\widetilde{x}(x, \omega), p(\psi))-\sigma_{L / D}(x(\omega), p(\psi)) \\
\text { s.t } x_{L} \leq x \leq x_{U}
\end{aligned}\right. \\
\text { SO-DO1 : }\left\{\begin{array}{cl}
\max _{x} L / D(x, p) \\
\text { s.t } & x_{L} \leq x \leq x_{U}
\end{array}\right.
\end{gathered}
$$
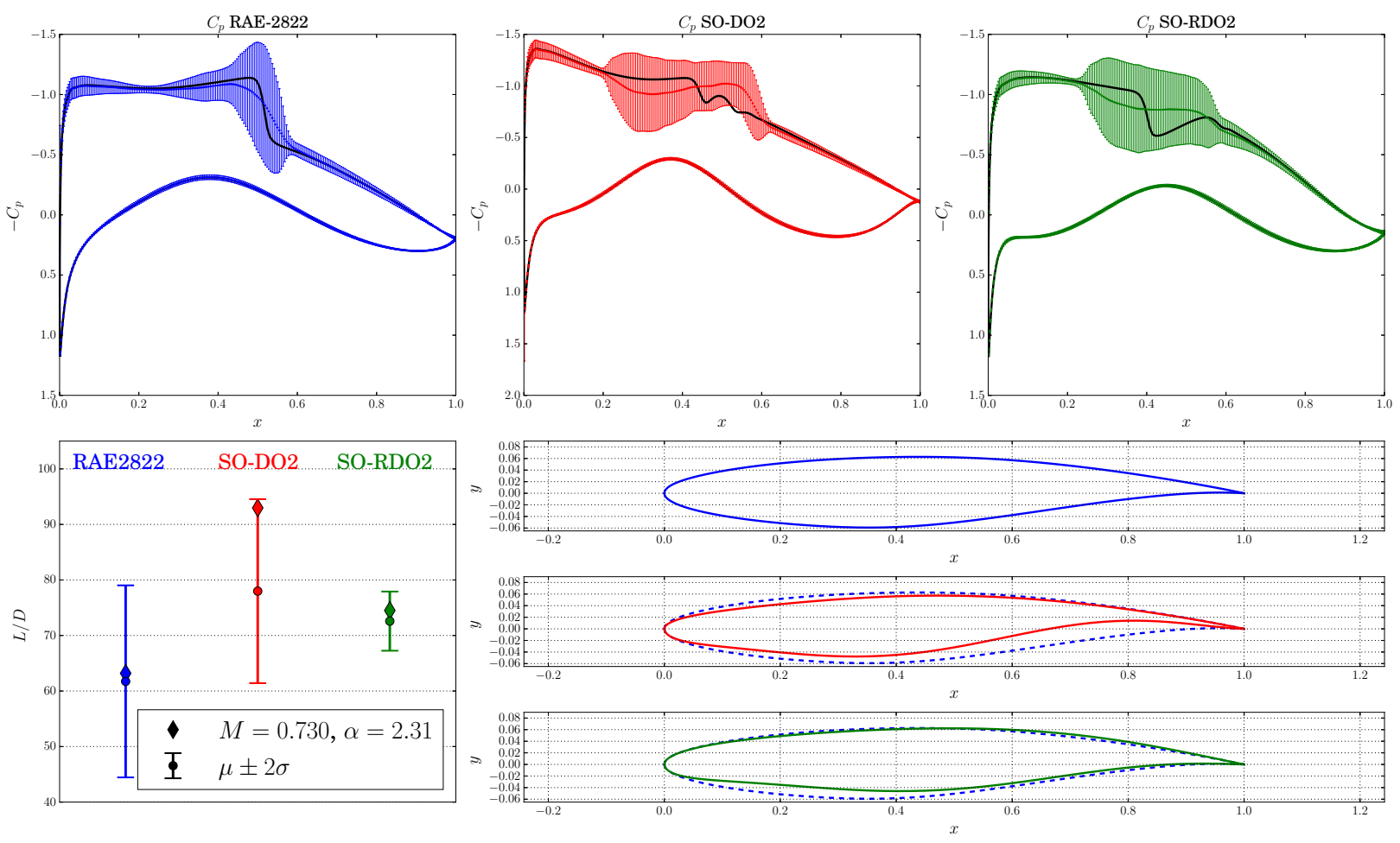

Figure 5: Performances of the SO-RDO2 and SO-DO2 airfoil compared with the original RAE-2822. The upper plots present the uncertain $C_{p}$ profile of the airfoils under operating and geometric uncertainties. The lower plots present the $L / D$ variation (mean \pm two standard deviations). The diamond symbol represents the performance of the airfoil at design condition (airfoil operating at Mach $=0.730, \alpha=2.31$ ).

In Figure 5 we present the result of the robust (SO-RDO2) and deterministic (SO-DO2) optimization of the $L / D$ ratio. As in the previous case we observe that the deterministically optimized airfoil is able to achieve the best performance at design condition however the $L / D$ ratio is highly sensitive to small variations of the geometry and operating conditions.

The robust airfoil is also in this case equipped with a relatively large leading edge. The quasi constant pressure plateau and the large aft chamber, compared to the SO-DO2, are capable of reducing the $L / D$ dispersion.

\section{V.B. Multi Objective Robust Design Optimization - Operating Uncertainties}

We now consider two competing robust objectives to be optimized simultaneously, namely minimize the drag coefficient $C_{D}$ and its dispersion and maximise the lift coefficient and its dispersion. We consider, first, only operating uncertainties (see Table 2) affecting the flow surrounding the airfoil and we use as design variables $x$ the Free Form Deformation (FFD) box coefficients (Figure 2(a)). 


$$
\text { MO-RDO1 : }\left\{\begin{aligned}
\underset{x}{\mathrm{P}-\min } & \left\{\mathcal{R}_{D}\left[C_{D}(x, p(\psi))\right], \mathcal{R}_{L}\left[C_{L}(x, p(\psi))\right]\right\} \\
\text { s.t } & x_{L} \leq x \leq x_{U}
\end{aligned}\right.
$$

with:

$$
\begin{aligned}
& \mathcal{R}_{D}\left[C_{D}(x, p(\psi))\right]=\mu_{C_{D}}(x, p(\psi))+\sigma_{C_{D}}(x, p(\psi)) \\
& \mathcal{R}_{L}\left[C_{L}(x, p(\psi))\right]=-\mu_{C_{L}}(x, p(\psi))+\sigma_{C_{L}}(x, p(\psi))
\end{aligned}
$$

We compare the results with the following multi objective optimization problem:

$$
\text { MO-DO1 : }\left\{\begin{aligned}
\underset{x}{\mathrm{P}-\min } & \left\{C_{D}(x, p), C_{L}(x, p)\right\} \\
\text { s.t } & x_{L} \leq x \leq x_{U}
\end{aligned}\right.
$$

In Figure 6 we present the result of MO-RDO1 and MO-DO1. For the candidate solution in the deterministic Pareto set (blue points, with lift coefficient higher and drag coefficient lower than the original RAE2822) and the RAE-2822 airfoil (black points) we perform an uncertainty analysis and compute the mean value (red square) and dispersion of the airfoils $C_{D}$ and $C_{L}$ (red ellipses correspond to two standard deviations) when they are operated in the uncertain environment. The blue stars represent candidates in the deterministic Pareto set that are very unstable when operating in the uncertain environment. Small variations in the angle of attack and Mach number lead to separated flow on the suction side of such airfoils.
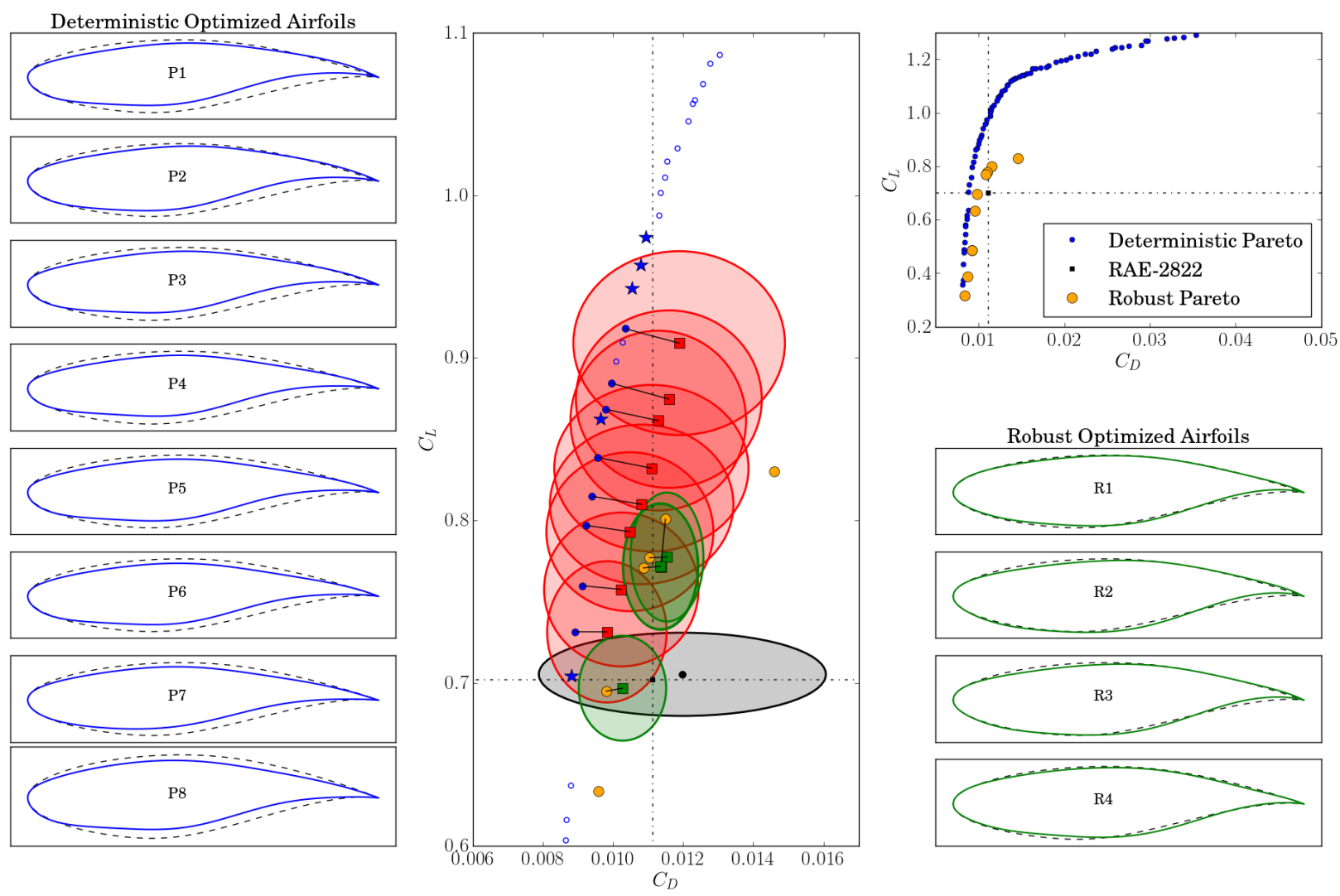

Figure 6: Deterministic and Robust Pareto fronts obtained by solving respectively the MO-DO1 and the MO-RDO1. The central plot is a blow-up view of the full Pareto. The red squares are the mean of $C_{L}$ and $C_{D}$ when the airfoils are operated in the uncertain environment, while the red ellipses are the dispersion around such mean values (two standard deviations). The green squares are the mean values of the robust optimal points and the green ellipses are their dispersion around such mean values (two standard deviations).

The first comment we can make regarding Figure 6 is that way fewer individuals populate the robust Pareto set with respect to the deterministic Pareto. Additionally we can observe a gathering of robust optimal 
candidates and their means around $C_{L} \approx 0.75$ and $C_{D} \approx 0.011$. Such robust candidates have quite similar performances and dispersion around the mean.

\section{V.C. Multi Objective Robust Design Optimization - Operating and Geometric Uncertainties}

Lastly we consider the multi objective problem of minimizing the drag coefficient $C_{D}$ and its dispersion and maximizing the lift coefficient and its dispersion with operating (system parameters $p(\psi)$, Mach number and angle of attack in Table 2) and geometric uncertainties at the same time (Table 2 and Table 3). In this set of simulations the design parameters $x$, are PARSEC parameters (Table 3 second column) and in the actual shape of the airfoil, uncertainty is added to those parameters.

$$
\text { MO-RDO2 : }\left\{\begin{array}{cl}
\underset{x}{\mathrm{P}-\min } & \left\{\mathcal{R}_{D}\left[C_{D}(\widetilde{x}(x, \omega), p(\psi))\right], \mathcal{R}_{L}\left[C_{L}(\widetilde{x}(x, \omega), p(\psi))\right]\right\} \\
\text { s.t } & x_{L} \leq x \leq x_{U}
\end{array}\right.
$$

with:

$$
\begin{aligned}
& \mathcal{R}_{D}\left[C_{D}(\widetilde{x}(x, \omega), p(\psi))\right]=\mu_{C_{D}}(\widetilde{x}(x, \omega), p(\psi))+\sigma_{C_{D}}(x, p(\psi)) \\
& \mathcal{R}_{L}\left[C_{L}(\widetilde{x}(x, \omega), p(\psi))\right]=-\mu_{C_{L}}(\widetilde{x}(x, \omega), p(\psi))+\sigma_{C_{L}}(\widetilde{x}(x, \omega), p(\psi))
\end{aligned}
$$

We compare the results with MO-DO1 but in this set of simulation we use the PARSEC coefficients as design parameters.

In Figure 7 we present the result of MO-RDO2 and MO-DO1. We perform, as for the previous case, an uncertainty analysis for the deterministic and robust candidates that dominate the original RAE-2822 (with lift coefficient higher and drag coefficient lower) and compute the mean value (red squares for the deterministic optimal and yellow squares for the robust optimal candidates) and dispersion of the airfoils $C_{D}$ and $C_{L}$ (red ellipses for the deterministic and green for robust candidates that correspond to two standard deviations) when they are operated in the uncertain environment. The blue stars represent candidates in the deterministic Pareto set that are very unstable when operating in the uncertain environment. Small variations in the geometry, angle of attack or Mach number lead to separated flow on the suction side of such airfoils.

As for the previous case we can observe a smaller population of candidates in the robust Pareto set with respect to the deterministic one. We can clearly identify, even better than in the previous case, a much higher stability of the performances of the robust optimized airfoils when operated in an uncertain environment and when affected by geometrical uncertainties.

\section{Conclusions}

In this work we have presented an extension of the Continuation Multi Level Monte Carlo (C-MLMC) to compute single and multi objective function based on mean and variance. We have described how to interface a well established Evolutionary Strategy algorithm, the CMA-ES, with the C-MLMC algorithm to perform robust design optimization. Additionally we provided parallel update formulas (the PQ-covariances) to compute the statistics of a quantity of interest and the statistical error contributions required to control the computational work and the accuracy of a C-MLMC analysis when the deterministic simulation (that are required in the stochastic analysis) are performed in parallel.

In the context aerodynamic shape design, we have described how to use the combination of CMA-ES and C-MLMC approach to perform robust optimization of transonic airfoils affected by a large number of operating and geometric uncertainties. We demonstrated with single and multi objective optimization problems that such methodology can be efficiently employed to design transonic airfoils that are less sensitive to those uncertainties. The technique has the potential to be extended to more complex problems where the stability and the reliability of the aerodynamic system is of crucial importance. Planned future work involves investigating and extending the C-MLMC approach to higher order moments, quantiles and coherent risk measures to perform robust and reliability based design optimization of aerodynamic systems.

\section{Acknowledgments}

This research has received funding from the EU-FP7 framework program within the project UMRIDA under grant agreement no. ACP3-GA-2013-605036. The authors also acknowledge CADMOS (Center for Advanced Modeling Science). 

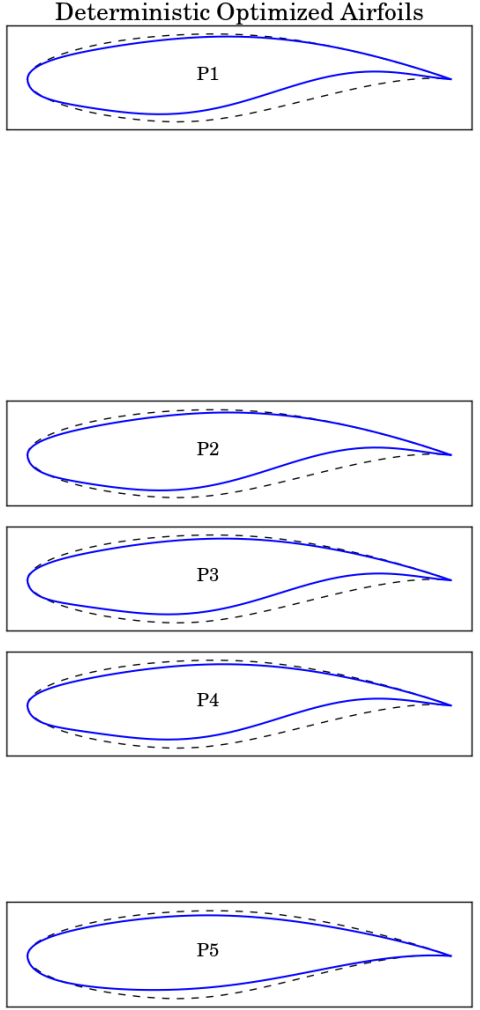
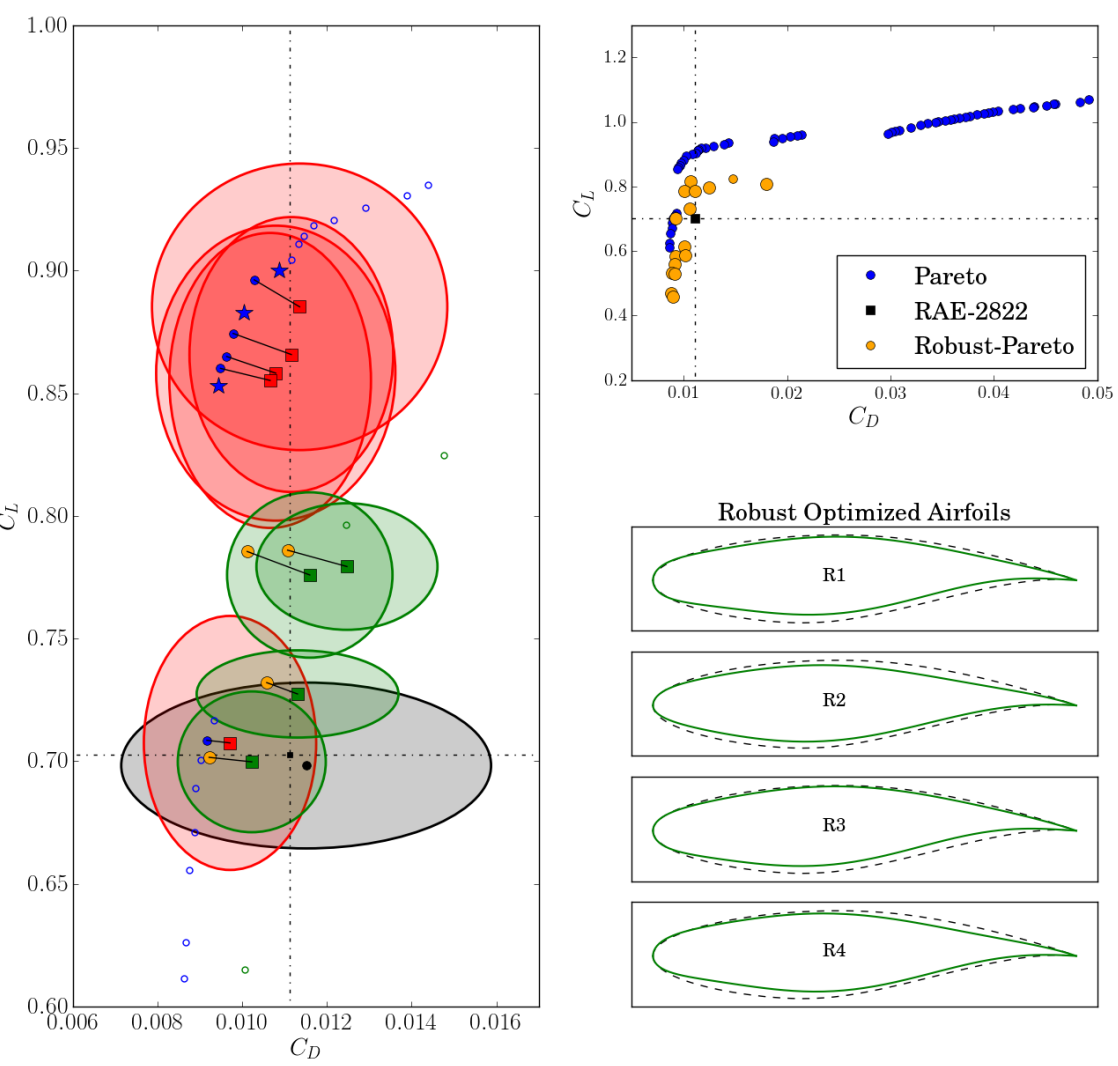

Robust Optimized Airfoils
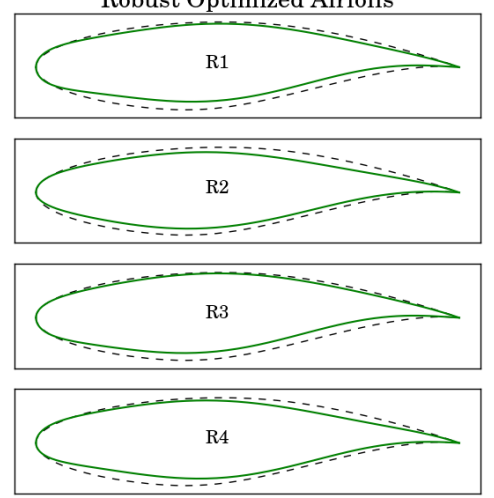

Figure 7: Deterministic and Robust Pareto fronts obtained by solving respectively the MO-DO1 (using PARSEC as design parameters) and the MO-RDO2. The central plot is a blow-up view of the full Pareto. he red squares are the mean of $C_{L}$ and $C_{D}$ when the airfoils are operated in the uncertain environment, while the red ellipses are the dispersion around such mean values (two standard deviations). The green squares are the mean values of the robust optimal points and the green ellipses are their dispersion around such mean values (two standard deviations). 


\section{A. Statistical Error for MLMC Variance Estimator}

We define here the following symbols:

$$
x^{\prime}=x-\mathbb{E}[x] \quad \bar{x}=\mathbb{E}^{\mathrm{MC}}[x]=\frac{1}{N} \sum_{i=1}^{N} x_{i} \quad \bar{x}^{\prime}=\frac{1}{N} \sum_{i=1}^{N}\left(x_{i}-\mathbb{E}[x]\right)
$$

The MSE for the MLMC estimator $\mathrm{V}^{\mathrm{MLMC}}\left[Q_{M}\right]$ for $\operatorname{Var}\left[Q_{M}\right]$ is

$$
\begin{aligned}
e\left(\mathrm{~V}^{\mathrm{MLMC}}\left[Q_{M}\right]\right)^{2}:= & \mathbb{E}\left[\left(\mathrm{V}^{\mathrm{MLMC}}\left[Q_{M}\right]-\operatorname{Var}[Q]\right)^{2}\right] \\
= & \underbrace{\left.\operatorname{Var}\left[Q_{M}\right]-\operatorname{Var}[Q]\right)^{2}}_{\left(\mathrm{B}-\mathrm{V}^{\mathrm{MLMC}}\right)}+\underbrace{\sum_{l=0}^{L} \mathbb{E}\left[\left(\mathrm{C}^{\mathrm{MC}}\left[X_{l}, Y_{l}\right]-\mathbb{C}\left[X_{l}, Y_{l}\right]\right)^{2}\right]}_{\left(\mathrm{SE}-\mathrm{V}^{\mathrm{MLMC} C}\right)} \\
\text { with } \quad & X_{l}=Q_{M_{l}}+Q_{M_{l-1}}, \quad Y_{l}=Q_{M_{l}}-Q_{M_{l}-1}, \\
& \mathrm{C}^{\mathrm{MC}}\left[X_{l}, Y_{l}\right]=\frac{1}{N_{l}-1} \sum_{i=1}^{N_{l}}\left(X_{i_{l}}-\mathbb{E}^{\mathrm{MC}}\left[X_{l}\right]\right)\left(Y_{i_{l}}-\mathbb{E}^{\mathrm{MC}}\left[Y_{l}\right]\right), \\
& \mathbb{C}\left[X_{l}, Y_{l}\right]=\mathbb{E}\left[\left(X_{l}-\mathbb{E}\left[X_{l}\right]\right)\left(Y_{l}-\mathbb{E}\left[Y_{l}\right]\right)\right] .
\end{aligned}
$$

We show here that the following are equivalent:

$$
\mathbb{E}\left[\left(\mathrm{C}^{\mathrm{MC}}\left[X_{l}, Y_{l}\right]-\mathbb{C}\left[X_{l}, Y_{l}\right]\right)^{2}\right]=\frac{1}{N_{l}}\left(\mathbb{E}\left[X_{l}^{2} Y_{l}^{2}\right]-\frac{N-2}{N-1} \mathbb{E}\left[X_{l} Y_{l}\right]^{2}+\frac{1}{N-1} \operatorname{Var}\left[X_{l}\right] \mathbb{V a r}\left[Y_{l}\right]\right)
$$


Proof. To shorten the notation we write in the following $X$ and $Y$ instead of $X_{l}$ and $Y_{l}$ (47):

$$
\begin{aligned}
& \mathbb{E}\left[\left(\mathrm{C}^{\mathrm{MC}}[X, Y]-\mathbb{C}[X, Y]\right)^{2}\right] \\
& =\mathbb{E}\left[\left(\frac{1}{N-1} \sum_{i=1}^{N}\left(X_{i}-\mathbb{E}^{\mathrm{MC}}[X]\right)\left(Y_{i}-\mathbb{E}^{\mathrm{MC}}[Y]\right)-\mathbb{E}[(X-\mathbb{E}[X])(Y-\mathbb{E}[Y])]\right)^{2}\right] \\
& =\mathbb{E}\left[\left(\frac{1}{N-1} \sum_{i=1}^{N}\left(X_{i} \pm \mathbb{E}[X]-\bar{X}\right)\left(Y_{i} \pm \mathbb{E}[Y]-\bar{Y}\right)-\mathbb{E}\left[X^{\prime} Y^{\prime}\right]\right)^{2}\right] \\
& =\mathbb{E}\left[\left(\frac{1}{N-1} \sum_{i=1}^{N}\left(X_{i}^{\prime}-\bar{X}^{\prime}\right)\left(Y_{i}^{\prime}-\bar{Y}\right)-\mathbb{E}\left[X^{\prime} Y^{\prime}\right]\right)^{2}\right] \\
& =\mathbb{E}\left[\left(\frac{1}{N-1}\left(\sum_{i=1}^{N} X_{i}^{\prime} Y_{i}^{\prime}-N \bar{X}^{\prime} \bar{Y}^{\prime}\right)-\mathbb{E}\left[X^{\prime} Y^{\prime}\right]\right)^{2}\right] \\
& =\mathbb{E}\left[\left(\frac{1}{N-1} \sum_{i=1}^{N} X_{i}^{\prime} Y_{i}^{\prime}-\frac{1}{N(N-1)} \sum_{j, k=1}^{N} X_{j}^{\prime} Y_{k}^{\prime}-\mathbb{E}\left[X^{\prime} Y^{\prime}\right]\right)^{2}\right] \\
& =\mathbb{E}\left[\left(\frac{1}{N} \sum_{i=1}^{N} X_{i}^{\prime} Y_{i}^{\prime}-\frac{1}{N(N-1)} \sum_{j \neq k}^{N} X_{j}^{\prime} Y_{k}^{\prime}-\mathbb{E}\left[X^{\prime} Y^{\prime}\right]\right)^{2}\right] \\
& =\mathbb{E}[\underbrace{\frac{1}{N^{2}}\left(\sum_{i=1}^{N} X_{i}^{\prime} Y_{i}^{\prime}\right)^{2}}_{(T 1)}+\underbrace{\frac{1}{N^{2}(N-1)^{2}}\left(\sum_{j \neq k}^{N} X_{j}^{\prime} Y_{k}^{\prime}\right)^{2}}_{(T 2)}+\underbrace{\mathbb{E}\left[X^{\prime} Y^{\prime}\right]^{2}}_{(T 3)} \\
& -\underbrace{\frac{2}{N^{2}(N-1)}\left(\sum_{i=1}^{N} X_{i}^{\prime} Y_{i}^{\prime}\right)\left(\sum_{j \neq k}^{N} X_{j}^{\prime} Y_{k}^{\prime}\right)}_{(T 4)}-\underbrace{\frac{2}{N}\left(\sum_{i=1}^{N} X_{i}^{\prime} Y_{i}^{\prime}\right) \mathbb{E}\left[X^{\prime} Y^{\prime}\right]}_{(T 5)} \\
& +\underbrace{\frac{2}{N(N-1)}\left(\sum_{j \neq k}^{N} X_{j}^{\prime} Y_{k}^{\prime}\right) \mathbb{E}\left[X^{\prime} Y^{\prime}\right]}_{(T 6)}]
\end{aligned}
$$


The terms in (49) can be rewritten as:

$$
\begin{aligned}
(T 1) & : \frac{1}{N^{2}} \mathbb{E}\left[\left(\sum_{i=1}^{N} X_{i}^{\prime} Y_{i}^{\prime}\right)^{2}\right]=\frac{1}{N^{2}} \mathbb{E}\left[\sum_{i, j=1}^{N} X_{i}^{\prime} Y_{i}^{\prime} X_{j}^{\prime} Y_{j}^{\prime}\right] \\
= & \frac{1}{N^{2}}\left(\mathbb{E}\left[\sum_{i=1}^{N}\left(X_{i}^{\prime}\right)^{2}\left(Y_{i}^{\prime}\right)^{2}\right]+\mathbb{E}\left[\sum_{i \neq j=1}^{N} X_{i}^{\prime} Y_{i}^{\prime} X_{j}^{\prime} Y_{j}^{\prime}\right]\right) \\
= & \frac{1}{N^{2}}\left(\mathbb{E}\left[\sum_{i=1}^{N}\left(X_{i}^{\prime} Y_{i}^{\prime}\right)^{2}\right]+\left(N^{2}-N\right) \mathbb{E}\left[X^{\prime} Y^{\prime}\right]^{2}\right) \\
= & \frac{1}{N} \mathbb{E}\left[\left(X^{\prime} Y^{\prime}\right)^{2}\right]+\frac{N-1}{N} \mathbb{E}\left[X^{\prime} Y^{\prime}\right]^{2} \\
(T 2) & : \frac{1}{N^{2}(N-1)^{2}} \mathbb{E}\left[\left(\sum_{j \neq k}^{N} X_{j}^{\prime} Y_{k}^{\prime}\right)^{2}\right]=\frac{1}{N^{2}(N-1)^{2}} \mathbb{E}\left[\sum_{j \neq k}^{N}\left(X_{j}^{\prime}\right)^{2}\left(Y_{k}^{\prime}\right)^{2}\right] \\
= & \frac{1}{N(N-1)} \mathbb{V a r}\left[X^{\prime}\right] \mathbb{V} a r\left[Y^{\prime}\right]+\frac{1}{N(N-1)} \mathbb{E}\left[X^{\prime} Y^{\prime}\right]^{2} \\
(T 3) & : \mathbb{E}\left[\mathbb{E}\left[X^{\prime} Y^{\prime}\right]^{2}\right]=\mathbb{E}\left[X^{\prime} Y^{\prime}\right]^{2} \\
(T 4) & : \frac{2}{N^{2}(N-1)} \mathbb{E}\left[\left(\sum_{i=1}^{N} X_{i}^{\prime} Y_{i}^{\prime}\right)\left(\sum_{j \neq k}^{N} X_{j}^{\prime} Y_{k}^{\prime}\right)\right]=0 \\
(T 5) & : \frac{2}{N} \mathbb{E}\left[\left(\sum_{i=1}^{N} X_{i}^{\prime} Y_{i}^{\prime}\right) \mathbb{E}\left[X^{\prime} Y^{\prime}\right]\right]=2 \mathbb{E}\left[X^{\prime} Y^{\prime}\right]^{2} \\
(T 6) & : \frac{2}{N(N-1)} \mathbb{E}\left[\left(\sum_{j \neq k}^{N} X_{j}^{\prime} Y_{k}^{\prime}\right) \mathbb{E}\left[X^{\prime} Y^{\prime}\right]=0\right. \\
&
\end{aligned}
$$

By substituting (50) in (49):

$$
\begin{aligned}
& \mathbb{E}\left[\left(\mathrm{C}^{\mathrm{MC}}[X, Y]-\mathbb{C}[X, Y]\right)^{2}\right] \\
= & \frac{1}{N} \mathbb{E}\left[\left(X^{\prime} Y^{\prime}\right)^{2}\right]+\frac{N-1}{N} \mathbb{E}\left[X^{\prime} Y^{\prime}\right]^{2}+\frac{1}{N(N-1)} \mathbb{V a r}\left[X^{\prime}\right] \mathbb{V a r}\left[Y^{\prime}\right] \\
& +\frac{1}{N(N-1)} \mathbb{E}\left[X^{\prime} Y^{\prime}\right]^{2}+\mathbb{E}\left[X^{\prime} Y^{\prime}\right]^{2}-2 \mathbb{E}\left[X^{\prime} Y^{\prime}\right]^{2} \\
= & \frac{1}{N}\left[\mathbb{E}\left[\left(X^{\prime} Y^{\prime}\right)^{2}\right]-\frac{N-2}{N-1} \mathbb{E}\left[X^{\prime} Y^{\prime}\right]^{2}+\frac{1}{N-1} \operatorname{Var}\left[X^{\prime}\right] \operatorname{Var}\left[Y^{\prime}\right]\right] .
\end{aligned}
$$

If we now consider the contribution of all levels in (47) we finally obtain:

$$
\begin{aligned}
e\left(\mathrm{~V}^{\mathrm{MLMC}}\left[Q_{M}\right]\right)^{2}: & =\underbrace{\mathbb{E}\left[\left(\mathrm{V}^{\mathrm{MLMC}}\left[Q_{M}\right]-\operatorname{Var}[Q]\right)^{2}\right]}_{\left(\mathrm{B}-\mathrm{V}^{\text {MLMC }}\right)} \\
& =\underbrace{\left(\operatorname{Var}\left[Q_{M}\right]-\mathbb{V} \operatorname{ar}[Q]\right)^{2}}_{\left(\mathrm{SE}-\mathrm{V}^{\text {MLMC }}\right)} \\
& +\underbrace{\sum_{l=0}^{L} \frac{1}{N_{l}}\left(\mathbb{E}\left[\left(X_{l}^{\prime} Y_{l}^{\prime}\right)^{2}\right]-\frac{N-2}{N-1} \mathbb{E}\left[X_{l}^{\prime} Y_{l}^{\prime}\right]^{2}+\frac{1}{N-1} \operatorname{Var}\left[X_{l}^{\prime}\right] \operatorname{Var}\left[Y_{l}^{\prime}\right]\right)}
\end{aligned}
$$




\section{B. Parallel update formulas for PQ-Covariances}

Let $S_{n}$ be an index set of cardinality $n$ and $\left\{\left(x_{i}, y_{i}\right), i \in S_{n}\right\}$ a collection of data. Our goal is to compute efficiently the quantity

$$
M_{p, q, S_{n}}=\sum_{i \in S_{n}}\left(x_{i}-\mu_{S_{n}}^{x}\right)^{p}\left(y_{i}-\mu_{S_{n}}^{y}\right)^{q} \quad \text { with } \quad \mu_{S_{n}}^{x}=\frac{1}{n} \sum_{i \in S_{n}} x_{i}, \quad \mu_{S_{n}}^{y}=\frac{1}{n} \sum_{i \in S_{n}} y_{i}
$$

Clearly

$$
M_{p, q, S_{n}}=\underbrace{\sum_{i \in S_{n_{1}}^{a}}\left(x_{i}-\mu_{S_{n}}^{x}\right)^{p}\left(y_{i}-\mu_{S_{n}}^{y}\right)^{q}}_{(A)}+\underbrace{\sum_{j \in S_{n_{2}}^{b}}\left(x_{j}-\mu_{S_{n}}^{x}\right)^{p}\left(y_{j}-\mu_{S_{n}}^{y}\right)^{q}}_{(B)}
$$

Consider now a partition $S_{n}=S_{n 1}^{a} \cup S_{n 2}^{b}, n_{1}+n_{2}=n$ and suppose that the quantity $M_{p, q, S_{n}^{a}}$ has already been computed. The question we address hereafter is how to compute efficiently $M_{p, q, S_{n}}$ using the previous computations.

For the sake of simplicity we denote from now on $S_{n_{1}}^{a}$ as $S^{a}, S_{n_{2}}^{a}$ as $S^{b}$ and we indicate with $\delta_{b, a}^{x}=-\delta_{a, b}^{x}=$ $\mu_{S^{b}}^{x}-\mu_{S^{a}}^{x}$ and with $\delta_{b, a}^{y}=-\delta_{a, b}^{y}=\mu_{S^{b}}^{y}-\mu_{S^{a}}^{y}$ :

$$
\begin{aligned}
& (A): \quad \sum_{i \in S^{a}}\left(x_{i}-\mu_{S}^{x}\right)^{p}\left(y_{i}-\mu_{S}^{y}\right)^{q} \\
& =\sum_{i \in S^{a}}\left(x_{i}-\frac{n_{1} \mu_{S^{a}}^{x}+n_{2} \mu_{S^{b}}^{x}}{n}\right)^{p}\left(y_{i}-\frac{n_{1} \mu_{S^{a}}^{y}+n_{2} \mu_{S^{b}}^{y}}{n}\right)^{q} \\
& =\sum_{i \in S^{a}}\left(x_{i}-\mu_{S^{a}}^{x}-\frac{n_{2}}{n}\left(\mu_{S^{b}}^{x}-\mu_{S^{a}}^{x}\right)\right)^{p}\left(y_{i}-\mu_{S^{a}}^{y}-\frac{n_{2}}{n}\left(\mu_{S^{b}}^{y}-\mu_{S^{a}}^{y}\right)\right)^{q} \\
& =\sum_{i \in S^{a}}\left[\sum_{k=0}^{p}\left(x_{i}-\mu_{S^{a}}^{x}\right)^{k}\left(-\frac{n_{2}}{n} \delta_{b, a}^{x}\right)^{p-k}\left(\begin{array}{l}
p \\
k
\end{array}\right)\right]\left[\sum_{l=0}^{q}\left(y_{i}-\mu_{S^{a}}^{y}\right)^{l}\left(-\frac{n_{2}}{n} \delta_{b, a}^{y}\right)^{q-l}\left(\begin{array}{l}
q \\
l
\end{array}\right)\right] \\
& =\sum_{i \in S^{a}} \sum_{k=0}^{p} \sum_{l=0}^{q}\left(x_{i}-\mu_{S^{a}}^{x}\right)^{k}\left(y_{i}-\mu_{S^{a}}^{y}\right)^{l}\left(-\frac{n_{2}}{n} \delta_{b, a}^{x}\right)^{p-k}\left(-\frac{n_{2}}{n} \delta_{b, a}^{y}\right)^{q-l}\left(\begin{array}{l}
p \\
k
\end{array}\right)\left(\begin{array}{l}
q \\
l
\end{array}\right) \\
& =\sum_{k=0}^{p} \sum_{l=0}^{q} \underbrace{\left(\sum_{i \in S^{a}}\left(x_{i}-\mu_{S^{a}}^{x}\right)^{k}\left(y_{i}-\mu_{S^{a}}^{y}\right)^{l}\right)}_{M_{k l, S^{a}}}\left(-\frac{n_{2}}{n} \delta_{b, a}^{x}\right)^{p-k}\left(-\frac{n_{2}}{n} \delta_{b, a}^{y}\right)^{q-l}\left(\begin{array}{l}
p \\
k
\end{array}\right)\left(\begin{array}{l}
q \\
l
\end{array}\right)
\end{aligned}
$$

The same procedure can be applied to term $(B)$ leading to:

$$
\begin{aligned}
(B): & \sum_{j \in S^{b}}\left(x_{i}-\mu_{S}^{x}\right)^{p}\left(y_{i}-\mu_{S}^{y}\right)^{q} \\
= & \sum_{j \in S^{b}} \sum_{k=0}^{p} \sum_{l=0}^{q}\left(x_{i}-\mu_{S^{b}}^{x}\right)^{k}\left(y_{i}-\mu_{S^{b}}^{y}\right)^{l}\left(-\frac{n_{1}}{n} \delta_{a, b}^{x}\right)^{p-k}\left(-\frac{n_{1}}{n} \delta_{a, b}^{y}\right)^{q-l}\left(\begin{array}{l}
p \\
k
\end{array}\right)\left(\begin{array}{l}
q \\
l
\end{array}\right) \\
= & \sum_{j \in S^{b}} \sum_{k=0}^{p} \sum_{l=0}^{q}\left(x_{i}-\mu_{S^{b}}^{x}\right)^{k}\left(y_{i}-\mu_{S^{b}}^{y}\right)^{l}\left(+\frac{n_{1}}{n} \delta_{b, a}^{x}\right)^{p-k}\left(+\frac{n_{1}}{n} \delta_{b, a}^{y}\right)^{q-l}\left(\begin{array}{l}
p \\
k
\end{array}\right)\left(\begin{array}{l}
q \\
l
\end{array}\right) \\
= & \sum_{k=0}^{p} \sum_{l=0}^{q} \underbrace{\left(\sum_{j \in S^{b}}\left(x_{i}-\mu_{S^{b}}^{x}\right)^{k}\left(y_{i}-\mu_{S^{b}}^{y}\right)^{l}\right)}_{M_{k l, S^{b}}}\left(+\frac{n_{1}}{n} \delta_{b, a}^{x}\right)^{p-k}\left(+\frac{n_{1}}{n} \delta_{b, a}^{y}\right)^{q-l}\left(\begin{array}{l}
p \\
k
\end{array}\right)\left(\begin{array}{l}
q \\
l
\end{array}\right)
\end{aligned}
$$


By substituting (55) and (56) in (54) we obtain:

$$
\begin{aligned}
M_{p, q, S_{n}} & =\sum_{k=0}^{p} \sum_{l=0}^{q}\left[M_{k, l, S^{a}}\left(-\frac{n_{2}}{n} \delta_{b, a}^{x}\right)^{p-k}\left(-\frac{n_{2}}{n} \delta_{b, a}^{y}\right)^{q-l}\left(\begin{array}{l}
p \\
k
\end{array}\right)\left(\begin{array}{l}
q \\
l
\end{array}\right)\right. \\
& \left.+M_{k, l, S^{b}}\left(+\frac{n_{1}}{n} \delta_{b, a}^{x}\right)^{p-k}\left(+\frac{n_{1}}{n} \delta_{b, a}^{y}\right)^{q-l}\left(\begin{array}{l}
p \\
k
\end{array}\right)\left(\begin{array}{l}
q \\
l
\end{array}\right)\right]
\end{aligned}
$$

For the sake of explanation we now reduce $S^{b}$ to a singleton:

$$
\begin{aligned}
& n_{2}=1, \quad S^{b}=\left\{x_{n}, y_{n}\right\}, \quad\left|S^{b}\right|=1 \\
& n_{1}=n-1, \quad S=S^{a} \cup\left\{x_{n}, y_{n}\right\}, \quad S^{a}=S_{n-1}, \quad\left|S^{a}\right|=n-1 \\
& \delta^{x}=x_{n}-\mu_{S_{n-1}}^{x}, \quad \delta^{y}=y_{n}-\mu_{S_{n-1}}^{y} .
\end{aligned}
$$

Since $M_{0,0, S_{n}}=n$ and $M_{1,0, S_{n}}=M_{0,1, S_{n}}=0$ we finally obtain a formula to estimate all the PQ-covariances:

\begin{tabular}{c}
$M_{p, q, S_{n}}=\left(\frac{n-1}{n}\right)^{p+q}\left(\delta_{x}\right)^{p}\left(\delta_{y}\right)^{q}\left(\frac{(-1)^{p+q}}{\left.(n-1)^{p+q-1}+1\right)}\right.$ \\
$\quad+\sum_{k=1}^{p} \sum_{l=1}^{q}\left[M_{k, l, n-1}\left(-\frac{\delta^{x}}{n}\right)^{p-k}\left(-\frac{\delta^{y}}{n}\right)^{q-l}\left(\begin{array}{l}p \\
k\end{array}\right)\left(\begin{array}{l}q \\
l\end{array}\right)\right]$ \\
$\quad+\sum_{k=2}^{p} M_{k, 0, n-1}\left(-\frac{\delta^{x}}{n}\right)^{p-k}\left(-\frac{\delta^{y}}{n}\right)^{q}\left(\begin{array}{c}p \\
k\end{array}\right)$ \\
\hline 1
\end{tabular}

\section{References}

\footnotetext{
${ }^{1}$ Hicks, R. M. and Vanderplaats, G. N., "Application of numerical optimization to the design of supercritical airfoils without drag-creep," Tech. rep., SAE Technical Paper, 1977.

${ }^{2}$ Tootkaboni, M., Asadpoure, A., and Guest, J. K., "Topology optimization of continuum structures under uncertainty-a polynomial chaos approach," Computer Methods in Applied Mechanics and Engineering, Vol. 201, 2012, pp. $263-275$.

${ }^{3}$ Dodson, M. and Parks, G. T., "Robust aerodynamic design optimization using polynomial chaos," Journal of Aircraft, Vol. 46, No. 2, 2009, pp. 635-646.

${ }^{4}$ Ho, S. and Yang, S., "A fast robust optimization methodology based on polynomial chaos and evolutionary algorithm for inverse problems," IEEE Transactions on Magnetics, Vol. 48, No. 2, 2012, pp. 259-262.

${ }^{5}$ Sankaran, S., Audet, C., and Marsden, A. L., "A method for stochastic constrained optimization using derivative-free surrogate pattern search and collocation," Journal of Computational Physics, Vol. 229, No. 12, 2010, pp. 4664-4682.
} 
${ }^{6}$ Simpson, T. W., Mauery, T. M., Korte, J. J., and Mistree, F., "Kriging models for global approximation in simulation-based multidisciplinary design optimization," AIAA journal, Vol. 39, No. 12, 2001, pp. 2233-2241.

${ }^{7}$ Heinrich, S., "Monte Carlo complexity of global solution of integral equations," Journal of Complexity, Vol. 14, No. 2, 1998, pp. 151-175.

${ }^{8}$ Giles, M. B., "Multilevel monte carlo path simulation," Operations Research, Vol. 56, No. 3, 2008, pp. $607-617$.

${ }^{9}$ Pisaroni, M., Nobile, F., and Leyland, P., "A Continuation Multi Level Monte Carlo (C-MLMC) Method for Uncertainty Quantifcation in Compressible Aerodynamics," Tech. rep., 2016.

${ }^{10}$ Collier, N., Haji-Ali, A.-L., Nobile, F., von Schwerin, E., and Tempone, R., "A continuation multilevel Monte Carlo algorithm," BIT Numerical Mathematics, 2014, pp. 1-34.

${ }^{11}$ Hansen, N. and Ostermeier, A., "Completely derandomized self-adaptation in evolution strategies," Evolutionary computation, Vol. 9, No. 2, 2001, pp. 159-195.

${ }^{12}$ Igel, C., Hansen, N., and Roth, S., "Covariance matrix adaptation for multi-objective optimization," Evolutionary computation, Vol. 15, No. 1, 2007, pp. 1-28.

${ }^{13}$ Pisaroni, M., Leyland, P., and Nobile, F., "A Multi Level Monte Carlo algorithm for the treatment of geometrical and operational uncertainties in internal and external aerodynamics," 46th AIAA Fluid Dynamics Conference, 2016, p. 4398.

${ }^{14}$ Bierig, C. and Chernov, A., "Convergence analysis of multilevel Monte Carlo variance estimators and application for random obstacle problems," Numerische Mathematik, Vol. 130, No. 4, 2015, pp. 579-613.

${ }^{15}$ Teckentrup, A., Scheichl, R., Giles, M., and Ullmann, E., "Further analysis of multilevel Monte Carlo methods for elliptic PDEs with random coefficients," Numerische Mathematik, Vol. 125, No. 3, 2013, pp. 569-600.

${ }^{16}$ Welford, B., "Note on a method for calculating corrected sums of squares and products," Technometrics, Vol. 4, No. 3, 1962, pp. 419-420.

${ }^{17}$ Chan, T. F., Golub, G. H., and LeVeque, R. J., "Updating formulae and a pairwise algorithm for computing sample variances," COMPSTAT 1982 5th Symposium held at Toulouse 1982, Springer, 1982, pp. 30-41.

${ }^{18}$ Pébay, P., "Formulas for robust, one-pass parallel computation of covariances and arbitrary-order statistical moments," Sandia Report SAND2008-6212, Sandia National Laboratories, 2008.

${ }^{19}$ Hansen, N., "The CMA evolution strategy: A tutorial," arXiv preprint arXiv:1604.00772, 2016.

${ }^{20}$ Drela, M., "A User's Guide to MSES 3.05," Massachusetts Institute of Technology (MIT), Cambridge, 2007. 